\title{
Titanium nitride-coated ceramic break for wall current monitors with an improved broadband frequency response
}

\author{
Yoshihiro Shobuda \\ JAEA, 2-4 Shirakata, Tokaimura, Nakagun, Ibaraki 319-1195, Japan \\ Takeshi Toyama \\ KEK, High Energy Accelerator Research Organization, 1-1 Oho, Tsukuba, Ibaraki 305-0801, Japan
}

(Received 25 June 2020; accepted 21 August 2020; published 10 September 2020)

\begin{abstract}
The beam impedance of a ceramic break with titanium nitride (TiN) coating consists of three electric components in parallel: resistive wall term caused by TiN, radiation term, and capacitive term made by the ceramic itself. The entire wall current continues to run in the thin TiN even when the skin depth is much larger than the chamber thickness, except for the extremely thin TiN satisfying the condition that the radiation loss from the ceramic break becomes lower than the energy loss due to the dc current on the thin TiN. This characteristic is useful in developing a wall current monitor with an improved frequency response. This study demonstrates the feature of the "ceramic break" monitor up to a few GHz from the theoretical and measurement points of view.
\end{abstract}

DOI: 10.1103/PhysRevAccelBeams.23.092801

\section{INTRODUCTION}

A short ceramic ring sandwiched by metal chambers is called a ceramic break. Ceramic breaks are often inserted between the chambers near bending magnets in proton synchrotrons to mitigate the eddy current effects on the metal chamber excited by the outside time-varying magnetic fields [1]. The inner surface of the ceramic is sometimes coated with titanium nitride (TiN) to suppress the secondary emission of electrons, caused by collisions between a part of the proton beams and the chamber surface. The TiN coating is beneficial in preventing a build-up of the electron cloud from destabilizing the beams [2-4].

The precise estimation of the beam impedances [5-7] is very important in realizing high-intensity beams in proton synchrotrons [8]; hence, the authors have carefully investigated the characteristic of the coupling impedances of several types of ceramic break: vacuum gap and the ceramic bream with and without the TiN coating [9-11]. We found that the impedance in the low frequency for a thin and short ceramic break is provided by the summation of three electric components in parallel, namely the resistive wall term caused by the TiN coating, radiation term, and capacitive term made by the ceramic itself [11].

Published by the American Physical Society under the terms of the Creative Commons Attribution 4.0 International license. Further distribution of this work must maintain attribution to the author(s) and the published article's title, journal citation, and DOI.
From an impedance point of view, the impedance becomes higher as the TiN thickness becomes thinner because the entire wall current continues to run in thin TiN even when the skin depth is much larger than the chamber thickness until the energy loss of the beam caused by the wall current through the TiN becomes larger than that of the radiation from the ceramic break $[10,11]$.

On the contrary, this characteristic of the ceramic break with a TiN coating can be used to monitor the longitudinal beam shapes, thanks to the improved frequency response under the condition that the impedance enhancement caused by the TiN is tolerable from a beam instability point of view. This is because the beam impedance of the ceramic break allows not to depend on the frequency significantly from very low to $\mathrm{GHz}$ region due to the thin TiN. This study mainly aims to demonstrate the distinction from the theoretical and measurement points of view.

Therefore, we fabricate a measurement setup, where the ceramic break is sandwiched by stainless steel chambers, inside of which an inner conductor made of copper is placed [7]. In this setup, we measure the longitudinal impedance of the ceramic break [7] to confirm whether the impedance follows the theoretically expected frequency behavior.

We then measure the voltage on the ceramic break by flowing a pulse current on the inner conductor, to see the relation between the voltage and the pulse current in the time domain. We also investigate the relation between the monitored signal on the ceramic break and the pulse current on the inner conductor by using a network analyzer in the frequency domain. We theoretically and experimentally 
associate the time domain analysis with the frequency domain analysis based on the electric circuit theory. Consequently, we find that the wall current monitor utilizing the ceramic break with the TiN coating has a better frequency characteristic of up to a few $\mathrm{GHz}$ and can be another candidate against the bunch shape monitor utilizing the triangle and concave pentagon electrodes, previously investigated by the authors [12], because the frequency response of the bunch shape monitor utilizing the electrodes at low frequencies maps the derivative of the bunch shape with time in the time domain, instead of the bunch shape itself.

Section II reviews the previous formula of the longitudinal impedance of a ceramic break with TiN coating [11]. Section III discusses the relationship between the monitored signal and the bunch shape. Section III A illustrates the measurement setup. Section III B presents the developed theoretical formulas for analysis of the measured data. Section III C explains the derived simple theoretical formulas describing the monitored pulse to comprehend the characteristic of the measured data. Section III D shows the measured longitudinal impedance of the ceramic break with TiN coating, voltage on the ceramic break, and original (beam) current pulse on the inner conductor. The paper is summarized in Sec. IV.

\section{IMPEDANCES OF CERAMIC BREAKS COATED WITH THIN TIN}

In Ref. [11], we derived the exact formulas of the longitudinal and transverse impedances of a ceramic break, whose inner surface is coated by thin TiN with a thickness $t$. In this section, we summarize the formula of the longitudinal impedance, where the thickness and the length of the ceramic break are sufficiently smaller than the inner radius of the ceramic break.

The parameters are specified as shown in Fig. 1, where cylindrical coordinates $(\rho, \theta, z)$ are used. The ceramic break consists of a ceramic with a relative dielectric constant $\epsilon^{\prime}$ and a relative permeability $\mu^{\prime}(=1)$ and a TiN with a conductivity $\sigma_{\mathrm{TiN}}$ The outer and inner radii of the ceramic break and those of the chambers of both sides are given in $a_{2}$ and $a$, respectively. The ceramic break is located in the $-w \leq z \leq w$ region (i.e., the length $g$ of the ceramic is equal to $2 w$ ), where the ceramic and the TiN are filled in the $a+t \leq \rho \leq a_{2}$ and $a \leq \rho<a+t$, respectively.

When the beam was assumed to have a cylindrically uniform density with a radius $\sigma$, and when the longitudinal

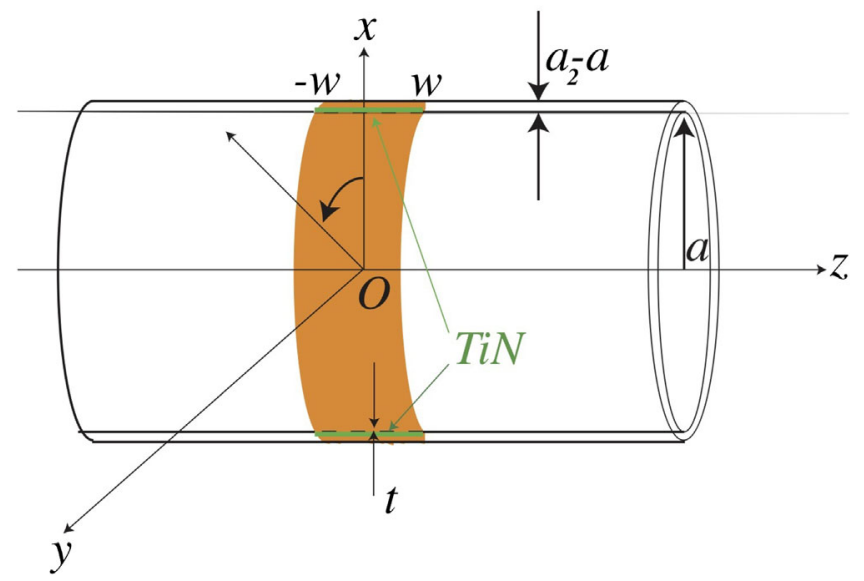

FIG. 1. Schematic of the ceramic break (orange object), sandwiched by perfectly conductive metal chambers. The inner and outer radii and length of the ceramic are denoted by $a+t, a_{2}$ and $g(=2 w)$, respectively. The inner surface of the ceramic is coated by a TiN (green) with thickness $t\left[\ll\left(a_{2}-a\right)\right]$.

impedance $Z_{L}$ is defined as the average of the longitudinal electric field (normalized by the beam current) over the beam cross-section, the longitudinal impedance of the short ceramic break can be well approximated as

$$
\begin{aligned}
Z_{L}= & -\frac{j Z_{0}}{k \beta \pi \sigma^{2}}\left(1-\frac{2 K_{0}(\bar{k} a) I_{1}^{2}(\bar{k} \sigma)}{I_{0}(\bar{k} a)}-2 I_{1}(\bar{k} \sigma) K_{1}(\bar{k} \sigma)\right) \mathcal{L}_{c} \\
& +Z_{\mathrm{cerTiN}, L}
\end{aligned}
$$

where $\mathcal{L}_{c}$ is the beam pipe length; $Z_{0}(=120 \pi \Omega)$ is the impedance of free space; $c$ is the light velocity; $\beta=v / c$; $v$ is the beam velocity; $\omega=2 \pi f ; f$ is the frequency; $k=\omega / c \beta ; I_{n}(z)$ and $K_{n}(z)$ are the modified Bessel functions [13]; $\gamma$ is Lorentz- $\gamma ; \bar{k}=k / \gamma$,

$$
V_{1}=\frac{-\frac{j k w \beta\langle Y(z)\rangle}{\pi Z_{0}} C_{0}^{(0)}\langle\alpha(z)\rangle-K_{0}^{(0)}\langle\alpha(z)\rangle}{\frac{I_{0}^{(0)}}{2 w}+\frac{K_{0}^{(0)} j k \beta\langle J(z)\rangle}{2 \pi Z_{0}}+\left(A_{0}^{(0)}+\frac{C_{0}^{(0)} j k \beta w\langle J(z)\rangle}{\pi Z_{0}}\right) \frac{j k \beta\langle Y(z)\rangle}{2 \pi Z_{0}}},
$$



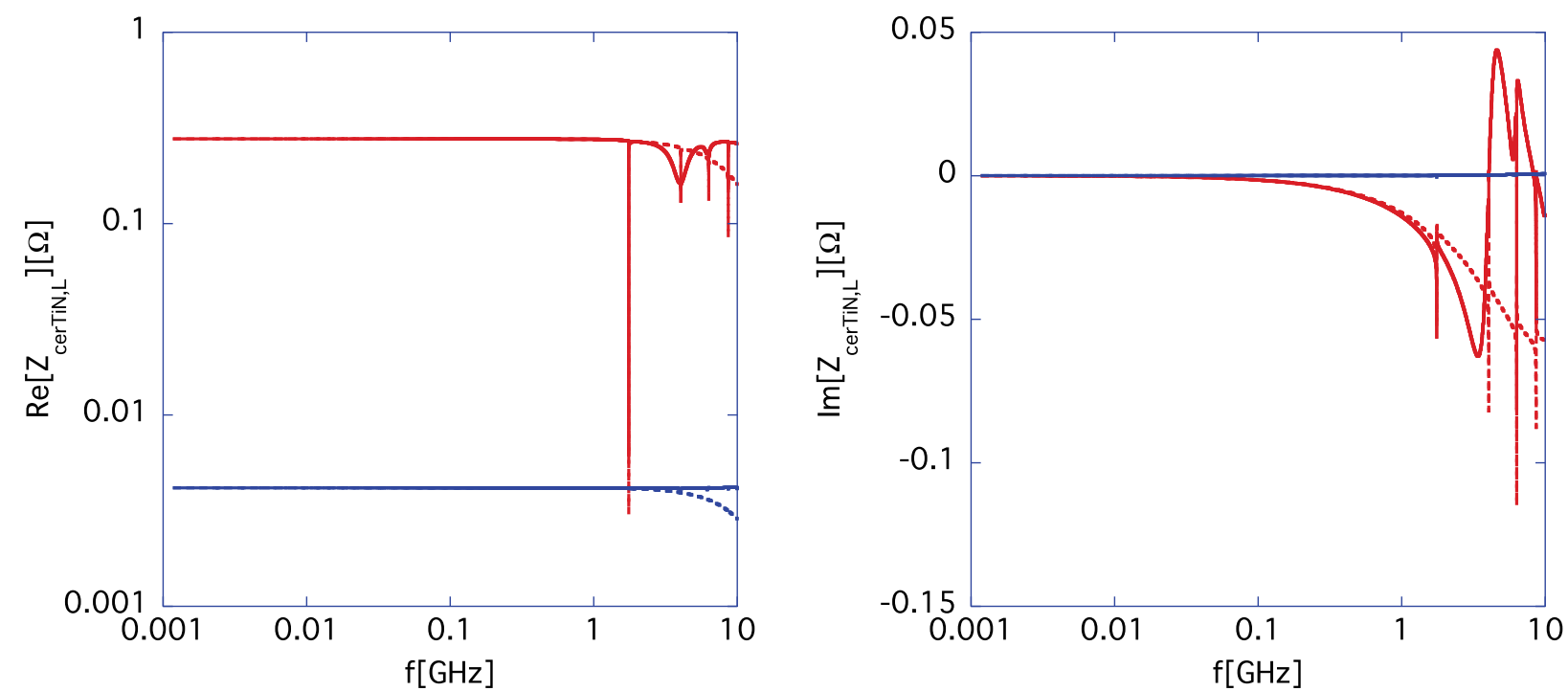

FIG. 2. Longitudinal impedances of the ceramic break with the TiN coating. The red and blue lines show the cases for $a=65 \mathrm{~mm}$, $a_{2}=70 \mathrm{~mm}, g=10 \mathrm{~mm}$, and $t=15 \mathrm{~nm}$ and for $a=65 \mathrm{~mm}, a_{2}=70 \mathrm{~mm}, g=10 \mathrm{~mm}$, and $t=1 \mu \mathrm{m}$, respectively. The solid and dashed lines depict the results by Eq. (2) and that by Eq. (5), respectively. In the right picture, the blue solid and the blue dashed lines are almost overlapped.

The coefficients $A_{0}^{(0)}, C_{0}^{(0)}, I_{0}^{(0)}, K_{0}^{(0)}, A_{0}^{(m)}, C_{0}^{(m)}, I_{0}^{(m)}$, and $K_{0}^{(m)}$ with positive integers $m$ and $n$, and the averages of functions $\langle\alpha(z)\rangle,\langle\langle\alpha(z)\rangle\rangle_{m},\langle J(z)\rangle,\langle\langle J(z)\rangle\rangle_{m},\left\langle\left\langle J^{(n)}(z)\right\rangle\right\rangle_{m}$, $\langle Y(z)\rangle,\langle\langle Y(z)\rangle\rangle_{m}$, and $\left\langle\left\langle Y^{(n)}(z)\right\rangle\right\rangle_{m}$ are given in Appendix. The first and the second terms $Z_{\text {cerTiN.L }}$ in Eq. (1) represent the nonrelativistic space charge impedance [9] and the coupling impedance of the ceramic break with TiN coating, respectively.

At low frequencies $(f<c / 2 \pi w)$, the longitudinal impedance is further approximated as follows

$Z_{\mathrm{cerTiN}, L} \simeq \frac{\frac{4 a_{2} I_{1}^{2}(\bar{k} \sigma)}{\sigma^{2} \bar{k}^{2} a I_{0}^{2}(\bar{k} a)}\left(\frac{\sin k w}{k w}\right)^{2}}{\frac{j k \beta a_{2}(\langle J(z)\rangle+\langle Y(z)\rangle)}{Z_{0}}-\frac{j \pi a_{2} \kappa_{\mathrm{TiN}} \tanh \kappa_{\mathrm{TiN}} t}{w k \beta Z_{0}}+\frac{j \omega \epsilon^{\prime} \pi a_{2}\left(a_{2}-a\right)}{c Z_{0} w}}$,

where

$$
\kappa_{\mathrm{TiN}}=\sqrt{j k \beta Z_{0} \sigma_{\mathrm{TiN}}} .
$$

The first term in the denominator of Eq. (5) represents the admittance of radiation effects out to free space as well as into the chamber, resulted from the conversion of a part of the wall-current when the beam passes through the ceramic break with TiN coating. The second term represents the resistive wall admittance caused by the TiN coating. The third term in the denominator is the ceramic break admittance, because the capacitance $C_{\text {cer }}$ caused by the ceramic break is approximated as

$$
C_{\text {cer }}=\frac{\epsilon^{\prime} \pi\left(a_{2}+a+t\right)\left(a_{2}-a-t\right)}{c Z_{0} 2 w} \simeq \frac{\epsilon^{\prime} \pi a_{2}\left(a_{2}-a\right)}{c Z_{0} w} .
$$

The longitudinal impedance of the ceramic break at low frequencies was approximately obtained by adding the following impedances in parallel: the impedance of the resistive wall caused by the TiN coating, that of the radiation, and that of the capacitor made by the ceramic.

Figure 2 compares the results by Eqs. (2) and (5) for two different sizes of ceramic breaks in the case of $\sigma_{\mathrm{TiN}}=5.88 \times 10^{6} \mathrm{~S} / \mathrm{m}$ and $\epsilon^{\prime}=11$. The left and right figures depict the real and imaginary parts of the impedances, respectively. The red and blue lines illustrate the cases for $a=65 \mathrm{~mm}, a_{2}=70 \mathrm{~mm}, g=10 \mathrm{~mm}$, and $t=15 \mathrm{~nm}$; and $a=65 \mathrm{~mm}, a_{2}=70 \mathrm{~mm}, g=10 \mathrm{~mm}$, and $t=1 \mu \mathrm{m}$, respectively. The solid and dashed lines represent the results by Eq. (2) and that by Eq. (5), respectively. Since both results converge at low frequencies, Eq. (5) gives a good approximation of the impedances there. The wall current continues to run on the thin insert, when the energy loss of the beam is smaller than when it converts to the radiation, or the displacement current in the ceramic. Consequently, the impedance does not depend on the frequency from the very low to $\mathrm{GHz}$ region where the skin depth is larger than the thickness of TiN, except for the ceramic break with extremely thin TiN coating as [10,11],

$$
t \ll\left(\frac{4 g}{\pi^{2} Z_{0}^{3} \sigma_{\text {TiN }}^{3}}\right)^{\frac{1}{4}}, \quad(\sim \text { typically of the order of a few ten } \mathrm{nm} \text { in the short ceramic break). }
$$


It indicates that nature tries to minimize the energy loss of the beam, determining whether the wall current continues to run on the thin insert, or converts to the radiation or the displacement current in the ceramic.

Note that straightforward numerical simulations are not suitable for calculating this kind of impedance, because extremely small mesh sizes are necessary (smaller than the thin TiN coating). Although we showed some simulation results partially including the thin TiN coating effect in Sec. III D, we will see the limit of this approach.

\section{RELATION BETWEEN THE BEAM PULSE SHAPE AND THE MONITORED SIGNAL ON THE CERAMIC BREAK}

In this section, we investigate how the beam pulse injected into the chambers is monitored through the ceramic break with the TiN coating by measurements, and how the original pulse can be reproduced by analyzing the frequency characteristic of the measurement setup (i.e., Eq. (51) summarizes the essence of the relation).

\section{A. Measurement setup}

Figure 3 represents two measurement setups, where the left and right pictures illustrate the setup in the time and frequency domains, respectively. In both pictures, the $10 \mathrm{~mm}$ long ceramic break with the TiN coating was sandwiched by $470 \mathrm{~mm}$ long stainless steel chambers with $a=65 \mathrm{~mm}$ and $a_{2}=70 \mathrm{~mm}$, whose ends were further sandwiched by two $242.85 \mathrm{~mm}$ tapers. The "electric impedance" of the ceramic break for the wall current is denoted by $Z_{\mathrm{TiN}}$. The inner conductor was placed at the center of the chambers. The setup was adjusted, such that

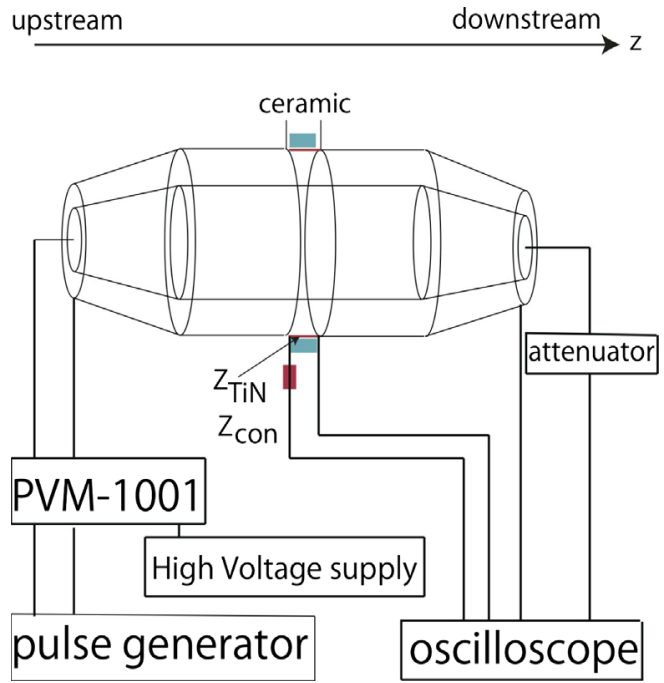

the inner and outer conductors make the coaxial structure, whose characteristic impedance is denoted by $Z_{50}(=50 \Omega)$ under the condition that the voltage standing wave ratio (VSWR) of the tapers is below 1.1 up to $1 \mathrm{GHz}$, so that reflections from the taper transitions have been minimized.

In the left picture, the upstream port on the taper was connected to the pulse generator [14], whose amplitude was enhanced by up to $1 \mathrm{kV}$ by the pulsed voltage module (PVM-1001 [15]) that required an external positive highvoltage power supply [16]. In the measurements, we generated a pulse with $5 \mathrm{~ns}$ rising and $5 \mathrm{~ns}$ falling times, thereby retaining a $60 \mathrm{~ns}$ flat top time. The downstream port on the taper was connected to the oscilloscope [17] through the attenuator [18]. A new port was created on the ceramic break by attaching the BNC connector. As the magnified picture in Fig. 3 shows, the TiN, both upstream and downstream chambers, and the inner/ground of the BNC connecters are electrically connected. The port on the ceramic break was connected to the oscilloscope such that we can observe the voltage on it. In this setup, we simulated the situation that the beam current monitored at the downstream port enters the upstream port of the chamber, and the wall current is monitored on the ceramic break.

In the right picture of Fig. 3, the upstream port of the chamber was terminated by the matched resistor with $Z_{50}$. The downstream port of the chamber was connected to the port (port 1) of the network analyzer [19], while the port on the ceramic break was connected to another port (port 2) of the network analyzer. This measurement aimed to precisely find the relation between the downstream port of the chamber and that of the port on the ceramic break in the frequency domain by using the network analyzer.

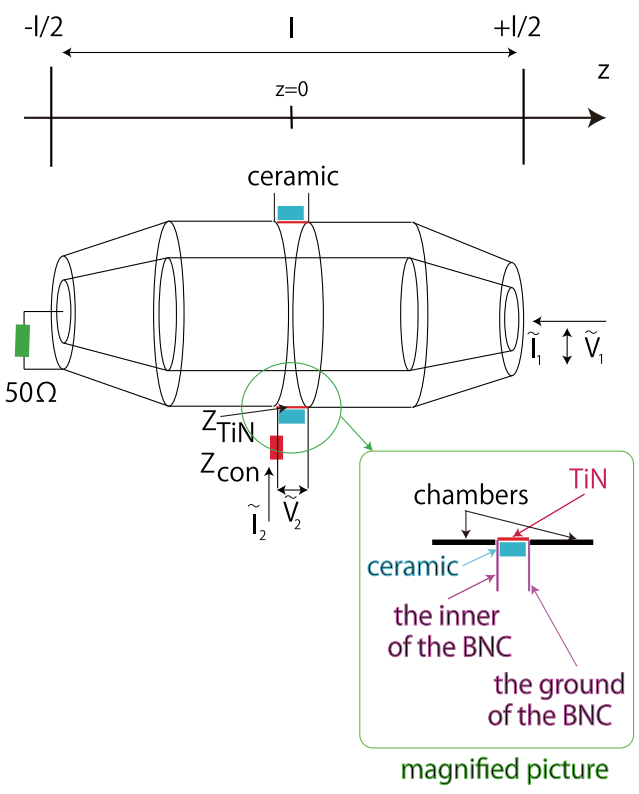

FIG. 3. Schematic of the measurement setups in the time domain (left) and the frequency domain (right), to observe the relation between the voltage on the ceramic break and the pulse current on the inner conductor. 

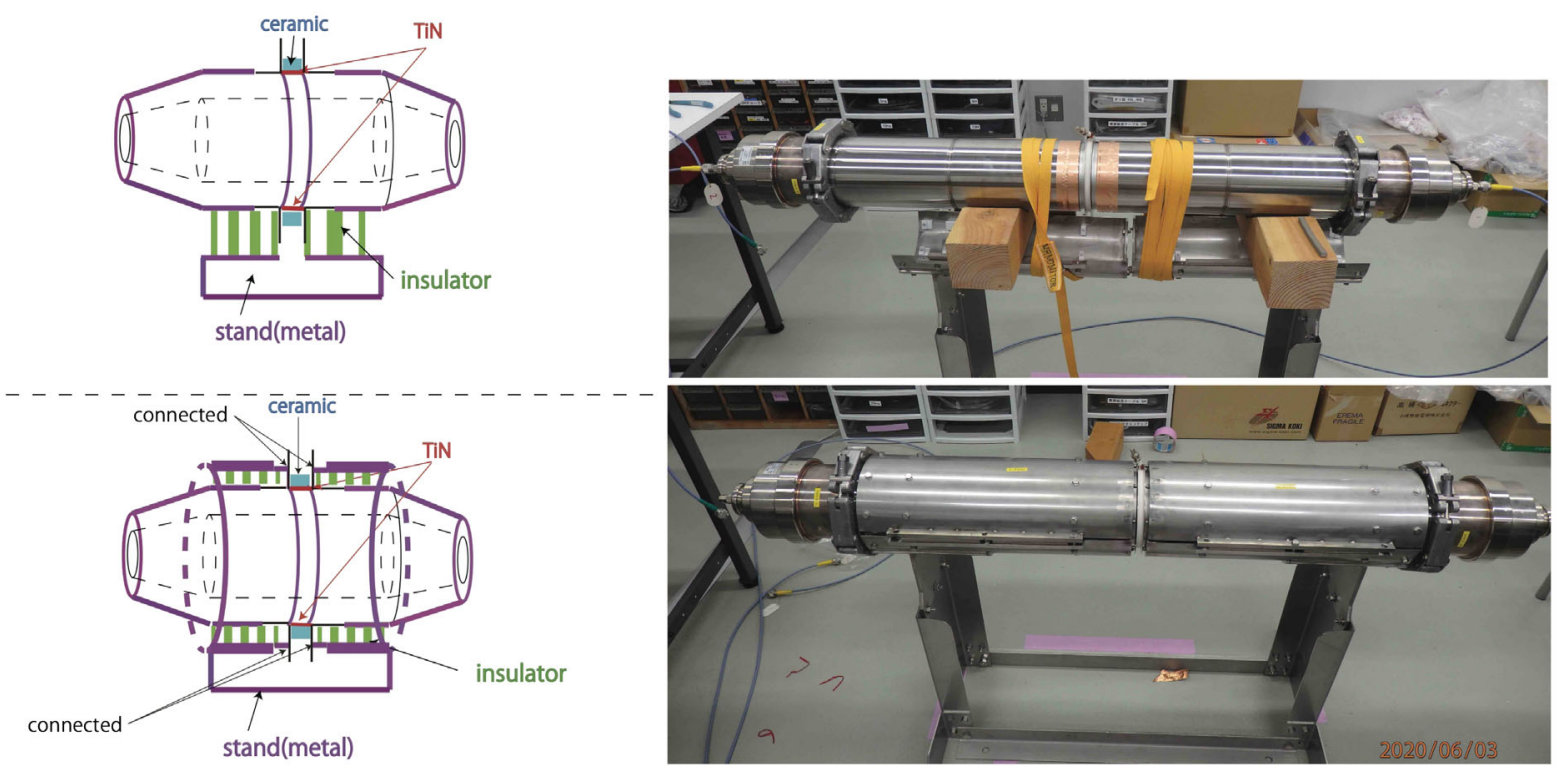

FIG. 4. Schematics (left) and the photos (right) of the measurement setups.

Let us investigate two types of ceramic breaks (upper and lower figures, Fig. 4) in advance to discuss the frequency characteristic of some monitored pulses on the ceramic break in the following section. The left pictures of Fig. 4 depict schematics of the ceramic breaks whose corresponding photos are shown in the right pictures. In the upper left/ right pictures, the ceramic break was supported by the insulator (green) on the metal stand, indicating that the wall current flowed only in TiN. In the lower left/right pictures, the edge of the ceramic break was 360 degrees surrounded and supported by conductive metal; hence, the wall current flowed in the TiN and the metal stand. Such differences of the wall current path can deform the shape of the monitored pulse for the respective ceramic breaks.

\section{B. Derivation of formulas for data analysis \\ 1. Derivation of log-formula}

First, let us review the standard wire method for the measurement of the longitudinal impedance $Z_{\text {cerTiN, } L}$ [7,20-22]. As shown in Fig. 5, the upstream and downstream ports were connected to ports 2 and 1 of the network analyzer, respectively. In the setup, the inner conductor was placed in the chambers, making the coaxial structure.

The voltages at ports 1 and 2 are given by $\tilde{V}_{1}$ and $\tilde{V}_{2}$ in the frequency domain, respectively. Meanwhile, the incoming currents from ports 1 and 2 are given by $\tilde{I}_{1}$ and $\tilde{I}_{2}$ in the frequency domain, respectively. They were divided by the incoming components $\tilde{V}_{1+}, \tilde{V}_{2+}, \tilde{I}_{1+}$, and $\tilde{I}_{2+}$ and the reflection components $\tilde{V}_{1-}, \tilde{V}_{2-}, \tilde{I}_{1-}$, and $\tilde{I}_{2-}$ as follows,

$$
\tilde{V}_{1}=\tilde{V}_{1+}+\tilde{V}_{1-}=\sqrt{Z_{50}}\left(\tilde{a}_{1}+\tilde{b}_{1}\right)
$$

$$
\begin{gathered}
\tilde{I}_{1}=\tilde{I}_{1+}-\tilde{I}_{1-}=\frac{\tilde{a}_{1}-\tilde{b}_{1}}{\sqrt{Z_{50}}}, \\
\tilde{V}_{2}=\tilde{V}_{2+}+\tilde{V}_{2-}=\sqrt{Z_{50}}\left(\tilde{a}_{2}+\tilde{b}_{2}\right), \\
\tilde{I}_{2}=\tilde{I}_{2+}-\tilde{I}_{2-}=\frac{\tilde{a}_{2}-\tilde{b}_{2}}{\sqrt{Z_{50}}},
\end{gathered}
$$

by introducing incident coefficients $\tilde{a}_{1}$ and $\tilde{a}_{2}$ and reflection coefficients $\tilde{b}_{1}$ and $\tilde{b}_{2}$.

The potential difference between the inner and outer conductors (chambers) and the current flow in the inner conductor are described as follows

$$
\Phi(z)-\Psi_{1}(z)=Z_{50}\left(\mathcal{A} e^{-j \omega \sqrt{L C} z}+\mathcal{B} e^{j \omega \sqrt{L C} z}\right),
$$
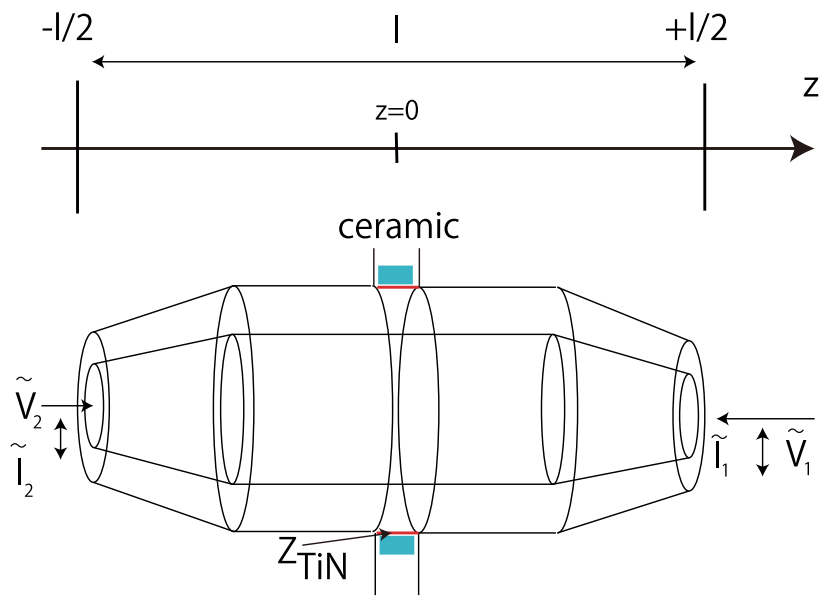

FIG. 5. Measurement setup for observing the beam impedance $Z_{\text {cerTiN }, L}$ or the electric impedance $Z_{\mathrm{TiN}}$. 


$$
I(z)=\mathcal{A} e^{-j \omega \sqrt{L C} z}-\mathcal{B} e^{j \omega \sqrt{L C} z}
$$

for $z<0$,

$$
\begin{gathered}
\Phi(z)-\Psi_{2}(z)=Z_{50}\left(\mathcal{C} e^{-j \omega \sqrt{L C} z}+\mathcal{D} e^{j \omega \sqrt{L C} z}\right), \\
I(z)=\mathcal{C} e^{-j \omega \sqrt{L C} z}-\mathcal{D} e^{j \omega \sqrt{L C} z},
\end{gathered}
$$

for $z>0$, where $L$ and $C$ are the inductance and the capacitance of the coaxial structure of the setup, respectively; $\Phi(z)$ is the potential of the inner conductor; $\Psi_{1}(z)$ and $\Psi_{2}(z)$ are the potentials of the outer conductor for $z<0$ and $z>0$, respectively; $I(z)$ is the current of the inner conductor; and $\mathcal{A}, \mathcal{B}, \mathcal{C}$, and $\mathcal{D}$ are the arbitrary coefficients determined by the boundary conditions. The potentials $\Phi(z), \Psi_{1}(z)$, and $\Psi_{2}(z)$, which are not used in the Ref. [20], play an important role in deriving the relation between the monitored signal on the ceramic break and the beam in the next subsection.

The boundary conditions on both sides of the chamber are given as

$$
\begin{gathered}
Z_{50}\left(\mathcal{A} e^{j \omega \sqrt{L C} \frac{l}{2}}+\mathcal{B} e^{-j \omega \sqrt{L C} \frac{l}{2}}\right)=\Phi\left(-\frac{l}{2}\right)-\Psi_{1}\left(-\frac{l}{2}\right)=\tilde{V}_{2}, \\
\mathcal{A} e^{j \omega \sqrt{L C} \frac{l}{2}}-\mathcal{B} e^{-j \omega \sqrt{L C} \frac{l}{2}}=\tilde{I}_{2}, \\
Z_{50}\left(\mathcal{C} e^{-j \omega \sqrt{L C} \frac{l}{2}}+\mathcal{D} e^{j \omega \sqrt{L C} \frac{l}{2}}\right)=\Phi\left(\frac{l}{2}\right)-\Psi_{2}\left(\frac{l}{2}\right)=\tilde{V}_{1}, \\
\mathcal{C} e^{-j \omega \sqrt{L C} \frac{l}{2}}-\mathcal{D} e^{j \omega \sqrt{L C} \frac{l}{2}}=-\tilde{I}_{1}, \\
\mathcal{A}-\mathcal{B}=\mathcal{C}-\mathcal{D}, \\
\Psi_{1}(0)+(\mathcal{A}-\mathcal{B}) Z_{\mathrm{TiN}}=\Psi_{2}(0),
\end{gathered}
$$

where $l$ is the total length of the chamber. Here, Eq. (22) is written as

$$
-Z_{50}(\mathcal{A}+\mathcal{B})+(\mathcal{A}-\mathcal{B}) Z_{\mathrm{TiN}}=-Z_{50}(\mathcal{C}+\mathcal{D}),
$$

by using Eqs. (13) and (15).

By solving Eqs. (17), (18), (19), (20), (21) and (23) for $\mathcal{A}, \mathcal{B}, \mathcal{C}, \mathcal{D}, \tilde{b}_{1}$, and $\tilde{b}_{2}$, the $S$-matrix is expressed as

$$
\begin{aligned}
& S_{11}=S_{22}=\frac{Z_{\mathrm{TiN}} e^{-j \omega \sqrt{L C} l}}{\left(2 Z_{50}+Z_{\mathrm{TiN}}\right)}, \\
& S_{12}=S_{21}=\frac{2 Z_{50} e^{-j \omega \sqrt{L C} l}}{\left(2 Z_{50}+Z_{\mathrm{TiN}}\right)} .
\end{aligned}
$$

The exponential factor $e^{j \omega \sqrt{L C l}}$ is typically determined by the other measurement after we replaced the ceramic break to the reference conductive pipe. That is, the $S_{12}^{(\text {ref })}$ component for the reference pipe is given by

$$
S_{12}^{(\mathrm{ref})}=\frac{1}{e^{j \omega \sqrt{L C l}}} .
$$

By combining Eq. (25) with Eq. (26), the electric impedance $Z_{\mathrm{TiN}}$ is expressed as

$$
Z_{\mathrm{TiN}}=2 Z_{50} \frac{\left(1-\frac{S_{12}}{S_{12}^{\text {(ref) }}}\right)}{\frac{S_{12}}{S_{12}^{\text {(ref) }}}} .
$$

Correspondingly, the beam impedance $Z_{\text {cerTiN }, L}$ is obtained by modifying Eq. (27) as [20,21]

$$
Z_{\text {cerTiN }, L}=-2 Z_{50} \log \left[\frac{S_{12}}{S_{12}^{(\text {ref })}}\right] .
$$

Another beam impedance measurement method, the resonant method, has an advantage that no matching is required in the measurement setup. However, the frequency resolution is limited due to the length of the setup, so we employ the standard wire method to measure the impedance [23].

\section{Relation between the monitored signal and the beam}

In this section, let us clarify the relation between the monitored signal on the ceramic break and the current pulse shape on the inner conductor by referring to Fig. 3. In the right figure of Fig. 3, the downstream end of the chamber and the port on the ceramic break are connected to ports 1 and 2 of the network analyzer, respectively. The boundary conditions caused by the upstream and downstream ends of the chamber provide

$$
\begin{gathered}
\tilde{V}_{1}=Z_{50}\left(\mathcal{C} e^{-j \omega \sqrt{L C} \frac{l}{2}}+\mathcal{D} e^{j \omega \sqrt{L C} \frac{l}{2}}\right), \\
\tilde{I}_{1}=-\mathcal{C} e^{-j \omega \sqrt{L C} \frac{l}{2}}+\mathcal{D} e^{j \omega \sqrt{L C} \frac{l}{2}} \\
\mathcal{C}-\mathcal{D}=\mathcal{A}-\mathcal{B}, \\
\mathcal{A}=0 .
\end{gathered}
$$

Moreover, the boundary condition at $z=0$ provides

$$
\begin{gathered}
\Psi_{1}(0)+Z_{\mathrm{con}} \tilde{I}_{2}-\tilde{V}_{2}=\Psi_{2}(0), \\
\Psi_{2}(0)-Z_{\mathrm{TiN}}\left(I(0)-\tilde{I}_{2}\right)=\Psi_{1}(0),
\end{gathered}
$$

where $Z_{\text {con }}$ is the accompanying impedance to electrically connect the ceramic break to port 2. Equations (33) and (34) are rewritten as

$$
-Z_{50}(\mathcal{A}+\mathcal{B})+Z_{\text {con }} I_{2}-V_{2}=-Z_{50}(\mathcal{C}+\mathcal{D}),
$$


$-Z_{50}(\mathcal{C}+\mathcal{D})-Z_{\mathrm{TiN}}\left(\mathcal{C}-\mathcal{D}-I_{2}\right)=-Z_{50}(\mathcal{A}+\mathcal{B})$,

by using Eqs. (13) and (15), respectively.

By solving Eqs. (29), (30), (31), (32), (35), and (36) for the coefficients $\mathcal{A}, \mathcal{B}, \mathcal{C}, \mathcal{D}, \tilde{b}_{1}$ and $\tilde{b}_{2}$, we obtain the scattering matrix $\left[\left(S_{11}^{(\text {mon })}, S_{12}^{(\text {mon })}\right),\left(S_{21}^{(\text {mon })}, S_{22}^{(\text {mon })}\right)\right]$ as

$S_{11}^{(\mathrm{mon})}=\frac{\left(Z_{50}+Z_{\mathrm{con}}\right) Z_{\mathrm{TiN}} e^{-j \omega \sqrt{L C l}}}{\left[2 Z_{\mathrm{TiN}} Z_{50}+\left(2 Z_{50}+Z_{\mathrm{TiN}}\right)\left(Z_{50}+Z_{\mathrm{con}}\right)\right]}$,

$S_{22}^{(\mathrm{mon})}=-\frac{\left[4 Z_{50}^{2}-\left(2 Z_{50}+Z_{\mathrm{TiN}}\right)\left(Z_{50}+Z_{\mathrm{con}}\right)\right]}{\left[2 Z_{\mathrm{TiN}} Z_{50}+\left(2 Z_{50}+Z_{\mathrm{TiN}}\right)\left(Z_{50}+Z_{\mathrm{con}}\right)\right]}$,

$S_{12}^{(\text {mon })}=S_{21}^{(\mathrm{mon})}=\frac{2 Z_{50} Z_{\mathrm{TiN}} e^{-j \omega \sqrt{L C} \frac{l}{2}}}{\left[2 Z_{\mathrm{TiN}} Z_{50}+\left(2 Z_{50}+Z_{\mathrm{TiN}}\right)\left(Z_{50}+Z_{\mathrm{con}}\right)\right]}$.

Reversely, the impedances $Z_{50}+Z_{\text {con }}$ and $Z_{\mathrm{TiN}}$ are expressed as

$$
\begin{gathered}
Z_{50}+Z_{\mathrm{con}}=\frac{2 Z_{50}\left(1-e^{j \omega \sqrt{L C} \frac{l}{2}} S_{12}^{(\mathrm{mon})}\right)}{1-S_{22}^{(\mathrm{mon})}}, \\
Z_{\mathrm{TiN}}=\frac{2 e^{j \omega \sqrt{L C} \frac{l}{2}} S_{12}^{(\mathrm{mon})} Z_{50}}{1-e^{j \omega \sqrt{L C} \frac{l}{2}} S_{12}^{\text {(mon) }}-S_{22}^{(\mathrm{mon})}},
\end{gathered}
$$

using the scattering matrix $\left[\left(S_{11}^{(\text {mon })}, S_{12}^{(\text {mon })}\right),\left(S_{21}^{(\text {mon })}\right.\right.$, $\left.\left.S_{22}^{(\text {mon })}\right)\right]$.

Now, let us consider the left picture in Fig. 3. In this case, the downstream end of the chamber and the port on the ceramic break are connected to ports 1 and 2 of the oscilloscope, respectively. After the observed signals in the time domain were Fourier-transformed, the boundary conditions can now be expressed as follows

$$
\begin{gathered}
\tilde{V}_{1}=Z_{50}\left(\mathcal{C} e^{-j \omega \sqrt{L C} \frac{l}{2}}+\mathcal{D} e^{j \omega \sqrt{L C} \frac{l}{2}}\right), \\
\tilde{I}_{1}=-\mathcal{C} e^{-j \omega \sqrt{L C \frac{l}{2}}}+\mathcal{D} e^{j \omega \sqrt{L C \frac{l}{2}}} \\
\mathcal{C}-\mathcal{D}=\mathcal{A}-\mathcal{B} \\
\mathcal{D}=0 \\
-Z_{50}(\mathcal{A}+\mathcal{B})+Z_{\text {con }} \tilde{I}_{2}+Z_{50} \tilde{I}_{2}=-Z_{50}(\mathcal{C}+\mathcal{D}), \\
-Z_{50}(\mathcal{C}+\mathcal{D})-Z_{\mathrm{TiN}}\left(\mathcal{C}-\mathcal{D}-\tilde{I}_{2}\right)=-Z_{50}(\mathcal{A}+\mathcal{B}) .
\end{gathered}
$$

In the frequency domain for $\mathcal{A}, \mathcal{B}, \mathcal{C}, \mathcal{D}, \tilde{V}_{1}$, and $\tilde{I}_{2}$, the relation between the voltage $\tilde{V}_{1}$ at port 1 and the monitored voltage $\tilde{V}_{2}$ at port 2 with the retarded effect is expressed as follows

$$
-\frac{e^{-j \omega \sqrt{L C} \frac{l}{2}} \tilde{V}_{2}}{\tilde{V}_{1}}=\frac{1}{Z_{50}} \mathcal{F},
$$

where

$$
\mathcal{F} \equiv \frac{Z_{\mathrm{TiN}} Z_{50}}{\left(Z_{\mathrm{TiN}}+Z_{50}+Z_{\mathrm{con}}\right)},
$$

$Z_{\text {con }}$ and $Z_{\mathrm{TiN}}$ are given by Eqs. (40) and (41), respectively, by using the network analyzer, and

$$
\tilde{V}_{2}=-Z_{50} \tilde{I}_{2} .
$$

Equation (48) is well approximated as

$$
-\frac{e^{-j \omega \sqrt{L C} \frac{l}{2} \tilde{V}_{2}}}{\tilde{V}_{1}} \simeq e^{j \omega \sqrt{L C} \frac{l}{2}} S_{12}^{(\mathrm{mon})},
$$

for $Z_{50} \gg Z_{\mathrm{TiN}}$ by referring to Eq. (39). The exponential factor and the minus sign in the left-hand side illustrate the time difference between the end of the chamber and the monitor position and the difference of the polarities between both signals, respectively.

Equation (51) reveals that $S_{12}^{\text {(mon) }}$ essentially relates the voltages at the downstream end of the chamber (original beam signal) and the port on the ceramic break (monitored signal), including the effect of the accompanying impedance $Z_{\text {con }}$ of port 2. In other words, the left-hand side of Eq. (51) is obtained by Fourier transforming the original current pulse and the monitored pulse measured with an oscilloscope, while the right-hand side of Eq. (51) is obtained using the network analyzer.

We use the following relation here,

$$
e^{j \omega \sqrt{L C} \frac{l}{2}}= \pm \frac{1}{\sqrt{S_{11}^{(\mathrm{thr})}+S_{12}^{(\mathrm{thr})}}},
$$

where the sign \pm is chosen such that the imaginary part of the argument of the exponential factor: $\omega \sqrt{L C} l / 2$ becomes positive for the positive frequency, when we calculate Eqs. (40), (41) and (51), instead of Eq. (26) derived by using the reference duct. Here, $\left[\left(S_{11}^{\text {(thr) }}, S_{12}^{\text {(thr) }}\right),\left(S_{21}^{(\text {thr })}, S_{22}^{\text {(thr) }}\right)\right]$ are the scattering matrix components, corresponding to the measurement setup in Fig. 6. Note that the terminal condition on the ceramic break in Fig. 6 is different from that in Fig. 5.

\section{Time domain approach}

As shown in Fig. 4, the ceramic break surroundings can affect on the electric impedance because the wall current path is modulated. This subsection considers how the trapezoidal beam pulse injected into the chamber is monitored through the ceramic breaks with TiN coating based on the previous electric circuit theory.

We already obtained the relation (48) between the current (beam) pulse on the inner conductor and the 


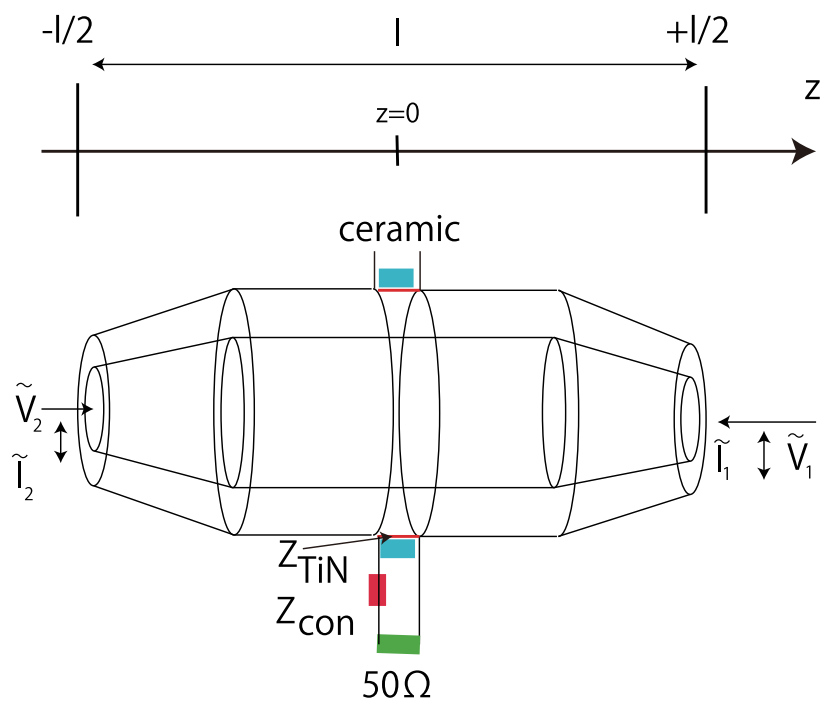

FIG. 6. Measurement setup to observe $\left[\left(S_{11}^{(\text {thr })}, S_{12}^{(\text {thr })}\right)\right.$, $\left.\left(S_{21}^{(\mathrm{thr})}, S_{22}^{(\mathrm{thr})}\right)\right]$.

monitored signal on the ceramic break in the frequency domain. Therefore, we can now survey the shape of the monitored signal in the time domain by applying the inverse Fourier transformation.

When the accompanying impedance $Z_{\text {con }}$ is negligible compared to $Z_{50}$, Eq. (48) is approximated as

$$
-e^{-j \omega \sqrt{L C} \frac{l}{2}} \tilde{V}_{2} \simeq \tilde{V}_{1} \frac{Z_{\mathrm{TiN}}}{Z_{50}}
$$

which is typically applicable at low frequencies. Equation (53) demonstrates the product of the beam pulse, and the impedance $Z_{\mathrm{TiN}}$ essentially determines the monitored shape of the signal.

Let us now apply the Fourier transform to Eq. (48) and see how the pulse behavior on the ceramic break is deformed in an analytical manner. For this purpose, we parametrized the factor $\mathcal{F}$, as

$$
\mathcal{F}=\frac{1}{\frac{1}{\mathcal{R}}+j \omega \mathcal{C}+\frac{1}{j \omega \mathcal{L}}}=\frac{\omega}{j \mathcal{C}\left(\omega^{2}-\frac{j}{\mathcal{C R}} \omega-\frac{1}{\mathcal{C L}}\right)},
$$

for general ceramic breaks.

The trapezoidal beam pulse $\mathcal{V}_{1}(t)$ shown as

$$
\begin{aligned}
\mathcal{V}_{1}(t)= & \frac{V_{0}}{t_{1}} t \Theta(t)-\frac{V_{0}}{t_{1}} t \Theta\left(t-t_{1}\right) \\
& +V_{0} \Theta\left(t-t_{1}\right)-V_{0} \Theta\left(t-t_{2}\right) \\
& -V_{0} \frac{\left(t-t_{3}\right)}{\left(t_{3}-t_{2}\right)} \Theta\left(t-t_{2}\right)+V_{0} \frac{\left(t-t_{3}\right)}{\left(t_{3}-t_{2}\right)} \Theta\left(t-t_{3}\right),
\end{aligned}
$$

where $\Theta(x)$ is the step function, produces its Fourier transformation $\tilde{V}_{1}$ as

$$
\tilde{V}_{1}=\int_{-\infty}^{\infty} d t e^{-j \omega t} \mathcal{V}_{1}(t)=\frac{\left(-e^{-j t_{2} \omega} t_{1}+e^{-j t_{3} \omega} t_{1}-t_{2}+e^{-j t_{1} \omega} t_{2}+t_{3}-e^{-j t_{1} \omega} t_{3}\right) V_{0}}{t_{1}\left(t_{2}-t_{3}\right) \omega^{2}}
$$

Subsequently, the product of $\tilde{V}_{1}$ and $\mathcal{F}$ corresponding to the monitored signal on the ceramic break is inverse Fouriertransformed as

$$
\mathcal{V}_{2}(t)=\int_{-\infty}^{\infty} d \omega \frac{e^{j \omega t}}{2 \pi} \tilde{V}_{1} \frac{\mathcal{F}}{Z_{50}}
$$

Finally, we obtain the analytical expression of the monitored signal as follows

$$
\begin{aligned}
\mathcal{V}_{2}(t)= & \int_{-\infty}^{\infty} d \omega \frac{V_{0}\left[-e^{-j\left(t_{2}-t\right) \omega} t_{1}+e^{-j\left(t_{3}-t\right) \omega} t_{1}-\left(t_{2}-t_{3}\right) e^{j \omega t}+e^{-j\left(t_{1}-t\right) \omega}\left(t_{2}-t_{3}\right)\right]}{2 \pi j Z_{50} \mathcal{C} t_{1}\left(t_{2}-t_{3}\right) \omega\left(\omega^{2}-\frac{j}{\mathcal{C} \mathcal{R}} \omega-\frac{1}{\mathcal{C} \mathcal{L}}\right)} \\
= & \left\{\begin{array}{l}
0, \quad \text { for } t \leq 0, \\
\frac{V_{0}}{Z_{50} \mathcal{C} t_{1}\left(\omega_{+}-\omega_{-}\right)}\left(\frac{1-e^{j \omega_{+} t}}{\omega_{+}}-\frac{1-e^{j \omega_{-}-t}}{\omega_{-}}\right), \quad \text { for } 0<t \leq t_{1}, \\
\frac{V_{0}}{Z_{50} \mathcal{C} t_{1}\left(\omega_{+}-\omega_{-}\right)}\left[\frac{e^{-j \omega_{+}\left(t_{1}-t\right)}-e^{j \omega_{+} t}}{\omega_{+}}+\frac{e^{j \omega_{-} t}-e^{-j \omega_{-}\left(t_{1}-t\right)}}{\omega_{-}}\right], \quad \text { for } t_{1}<t \leq t_{2}, \\
\frac{V_{0}}{Z_{50} \mathcal{C} t_{1}\left(t_{2}-t_{3}\right)\left(\omega_{+}-\omega_{-}\right)}\left[\left(\frac{1-e^{-j \omega_{+}\left(t_{2}-t\right)}}{\omega_{+}}+\frac{e^{-j \omega_{-}\left(t_{-}-t\right)}-1}{\omega_{-}}\right) t_{1}\right. \\
\left.+\left(\frac{e^{-j \omega_{+}\left(t_{1}-t\right)}-e^{j \omega_{+} t}}{\omega_{+}}+\frac{e^{j \omega_{-}-e_{-}}}{\omega_{-}}\right)\left(t_{2}-t_{3}\right)\right], \quad \text { for } t_{2}<t \leq t_{3}, \\
\frac{V_{0}}{Z_{50} \mathcal{C} t_{1}\left(t_{2}-t_{3}\right)\left(\omega_{+}-\omega_{-}\right)}\left[\left(\frac{e^{-j \omega_{+}\left(t_{3}-t\right)}-e^{-j \omega_{+}\left(t_{2}-t\right)}}{\omega_{+}}+\frac{e^{-j \omega_{-}\left(t_{2}-t\right)}-e^{-j \omega_{-}\left(t_{3}-t\right)}}{\omega_{-}}\right) t_{1}\right. \\
\left.+\left(\frac{e^{-j \omega_{+}\left(t_{1}-t\right)}-e^{j \omega_{+} t}}{\omega_{+}}+\frac{e^{j \omega_{-} t}-e^{-j \omega_{-}\left(t_{1}-t\right)}}{\omega_{-}}\right)\left(t_{2}-t_{3}\right)\right], \quad \text { for } t_{3} \leq t,
\end{array}\right.
\end{aligned}
$$


where

$$
\omega_{ \pm}=\frac{j \mathcal{L} \pm \sqrt{-\mathcal{L}^{2}+4 \mathcal{L C} \mathcal{R}^{2}}}{2 \mathcal{C} \mathcal{L} \mathcal{R}}
$$

The above general expression is simplified as

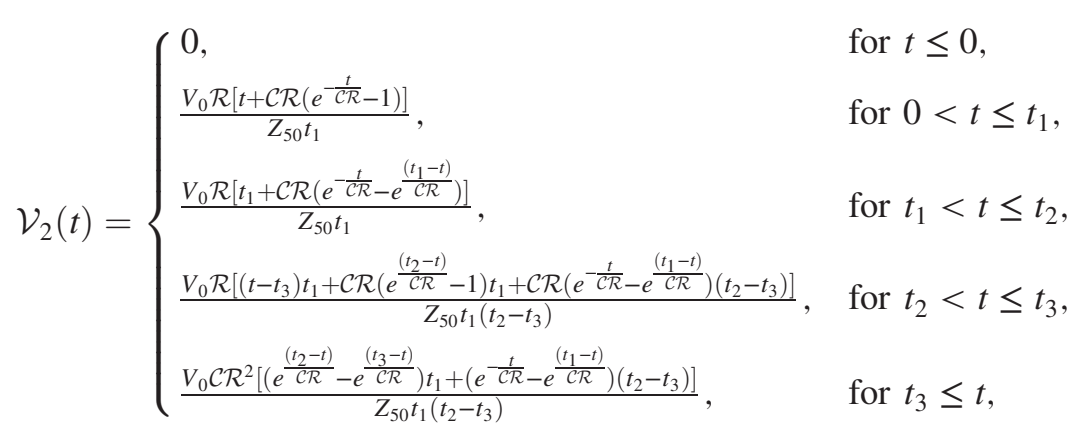

for $\mathcal{L}=\infty$ (i.e., capacitive part dominant impedance, although this does not occur in most realistic ceramic breaks). Meanwhile,

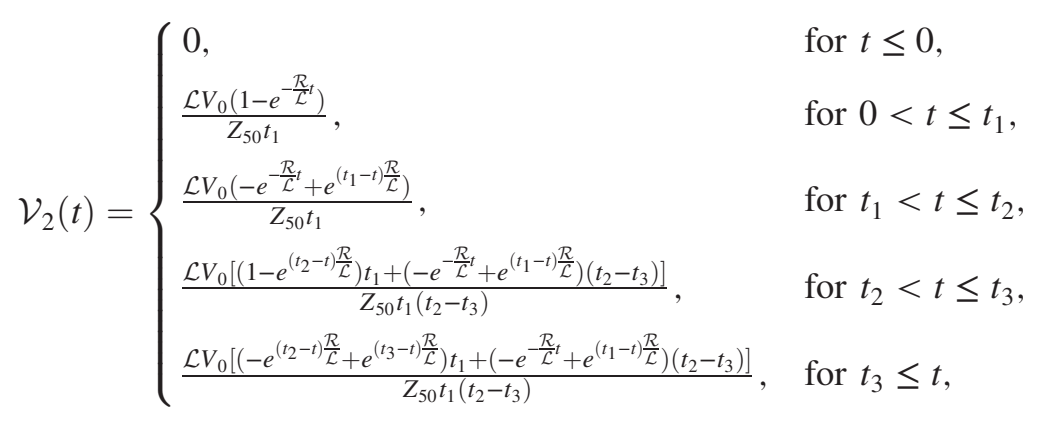

for $\mathcal{C}=0$ (i.e., inductive part dominant impedance).

\section{Measurements}

Let us now measure the longitudinal impedance $Z_{\text {cerTiN }, L}$ for the ceramic break ( $a=65 \mathrm{~mm}$ and $g=10 \mathrm{~mm}$ ) with TiN coating in the upper pictures of Fig. 4. The ceramic break is supported by the insulator and all wall current flows on TiN. Figure 7 shows the measurement (left) and theoretical (right) results, where the TiN thickness $(t)$ is assumed to be $10 \mathrm{~nm}$, and its conductivity is $\sigma_{\mathrm{TiN}}=$ $3.5 \times 10^{6}[\mathrm{~S} / \mathrm{m}]$ in the theoretical estimation to produce the dc resistance of the ceramic break.

The left picture illustrates the longitudinal impedance $Z_{\text {cerTiN, } L}$ (red/blue) and the electric impedance $Z_{\mathrm{TiN}}$ (black/ green), following Eqs. (28) and (27), respectively. The red and black lines show the real part, while the blue and green lines depict the imaginary part of the impedances. The difference between both experimental results was negligible. This is because the log-formula (28) reduces to Eq. (27), when the dimension of the ceramic break is smaller than the wave length. Hence, we confirm that the frequency characteristic of the beam impedance is identical to that of the electric impedance. Moreover, the theoretical results explain the measurements, except for the oscillation observed at a high frequency.

The oscillation behavior at the high frequency is expected to be contributed from the systematic error caused by the impedance mismatch between the chambers sandwiching the ceramic break and their outside tapers.

Let us partially rely on the simulation of the ceramic break with TiN coating by using MicroWave Solver of CST [24]. The theoretical and measurement results suggest that the surface impedance $Z_{s}$ on TiN is approximated as a dc resistance. Under this assumption, we may use "tabulated surface impedance" in one layer and one-dimensional model by using the CST [24] with the conductivity of $\sigma_{\mathrm{TiN}}$ and the thickness of $\operatorname{TiN}(t)$ as the input parameters.

The longitudinal electric field $E_{z}$ and the azimuthal magnetic field $H_{\theta}$ are related as follows

$$
E_{z}=Z_{s} H_{\theta},
$$

via the surface impedance $Z_{s}$. When the "width-to-height ratio" of the cross section of TiN is set to 10 (default), not $2 \pi a / t$, in the tabulated surface impedance model in the CST and when the electro-magnetic fields are assumed to 

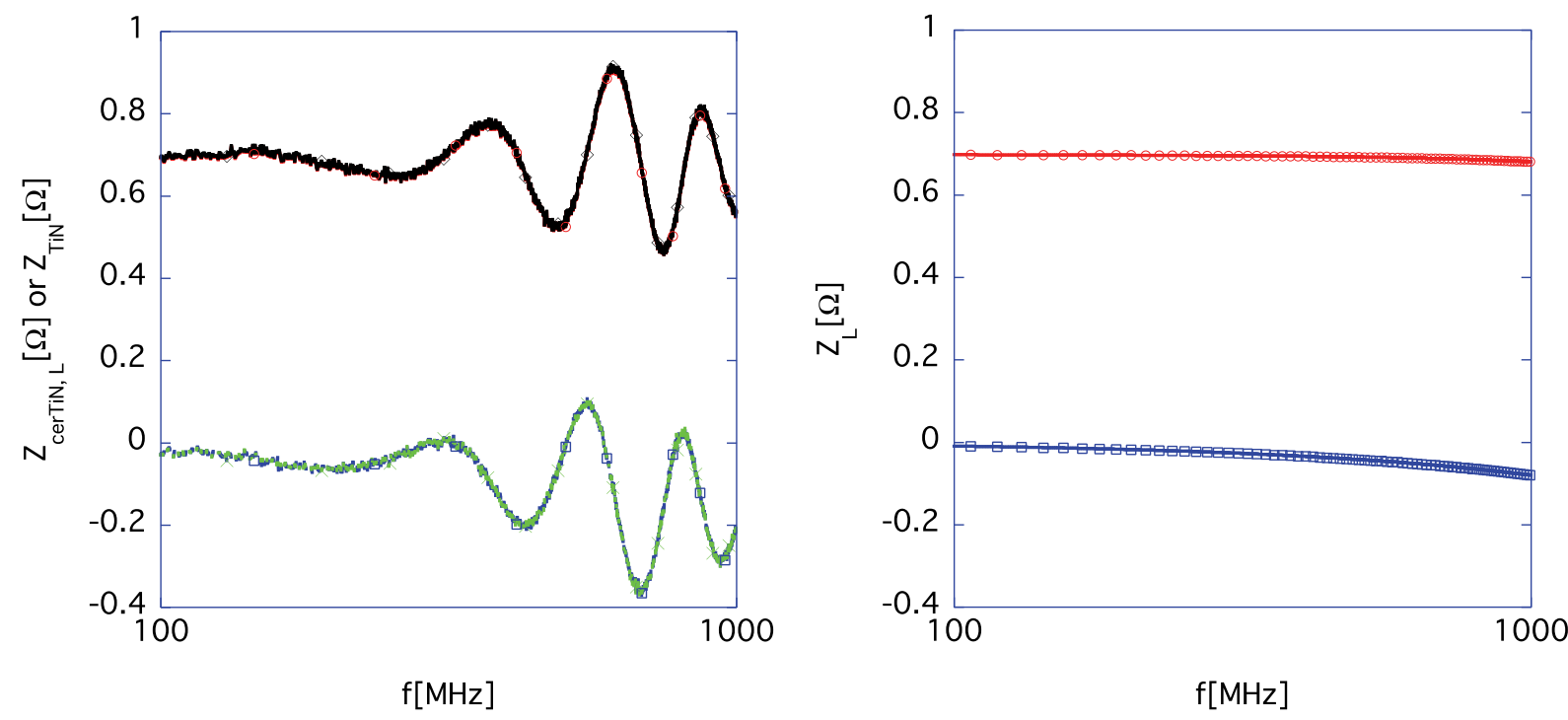

FIG. 7. Measurements (left) and theoretical (right) results of the longitudinal impedance. The longitudinal impedance $Z_{\text {cerTiN }, L}$ (red/ blue) and the electric impedance $Z_{\mathrm{TiN}}$ (black/green) are shown overlapped in the left picture. The horizontal axis is frequency in logarithmic scale.

be constant in space, the dc resistance $R_{\mathrm{DC}}$ of TiN is described as

$$
R_{\mathrm{DC}}=\frac{Z_{s} g}{2(t+10 t)}
$$

after the application of Ampere's law for Eq. (62), which is equivalent to

$$
R_{\mathrm{DC}}=\frac{g}{\sigma_{\mathrm{TiN}} 10 t^{2}} .
$$

Hence, the surface impedance is automatically calculated in the CST as

$$
Z_{s}=\frac{11}{\sigma_{\mathrm{TiN}} 5 t},
$$

by using the conductivity of $\sigma_{\mathrm{TiN}}$ and the thickness of TiN $(t)$ as the input parameters.

First, let us neglect the tapers. Both ends of the chambers are terminated with $50 \Omega$ waveguide ports. Figure 8 illustrates the simulation results, where the red and blue lines show the real and imaginary parts of the impedance, respectively. The real part of the impedance was constant, as expected, although it was 1.85 times larger than that in the theoretical and measurement results. One possible reason for this discrepancy can be a limit of one layer one-dimensional tabulated surface impedance model to estimate the real part of the impedance. The imaginary part of the impedance seemed to become slightly capacitive because of the ceramic effect. However, note that the tabulated surface impedance model produces an 'opaque' material. Accordingly, in principle, the effect outside of the
TiN cannot be included in the simulation. In this sense, it would be better to focus on the real part of the impedance rather than its imaginary part.

With the recognition of the lack of accuracy of the simulation results, let us try to include the effect of the tapers on the simulations, where both ends of the tapers are terminated with $50 \Omega$ waveguide ports. The tapers are modeled to satisfy the condition that the VSWR of the

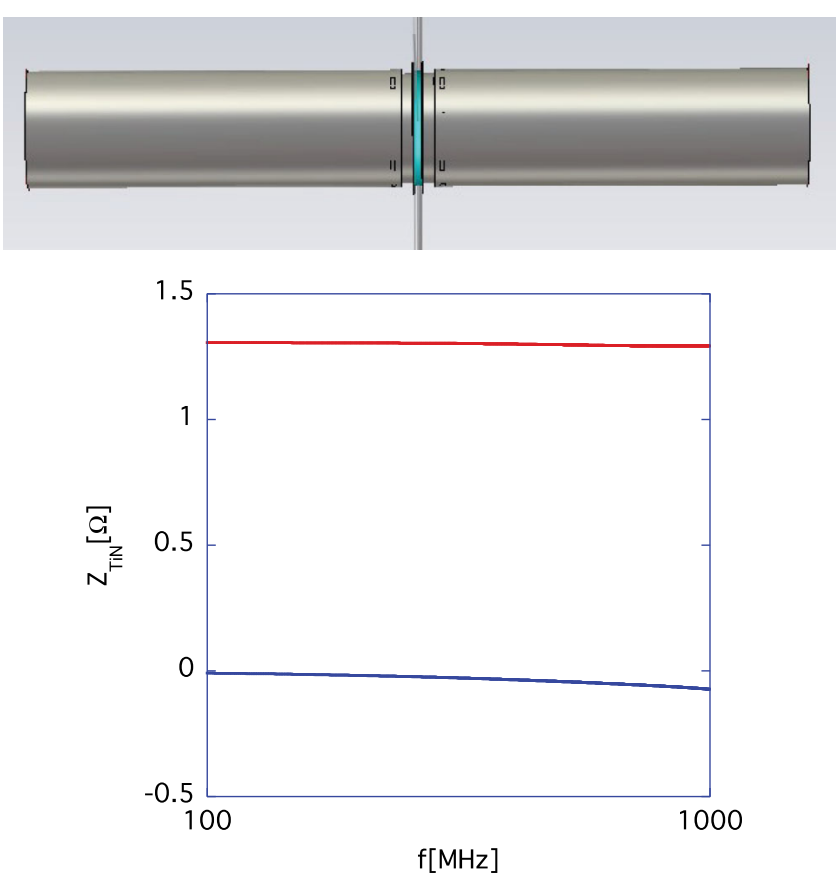

FIG. 8. Simulations (lower) of the longitudinal impedance without tapers in the setup shown in the upper figure. The horizontal axis is frequency in logarithmic scale. 

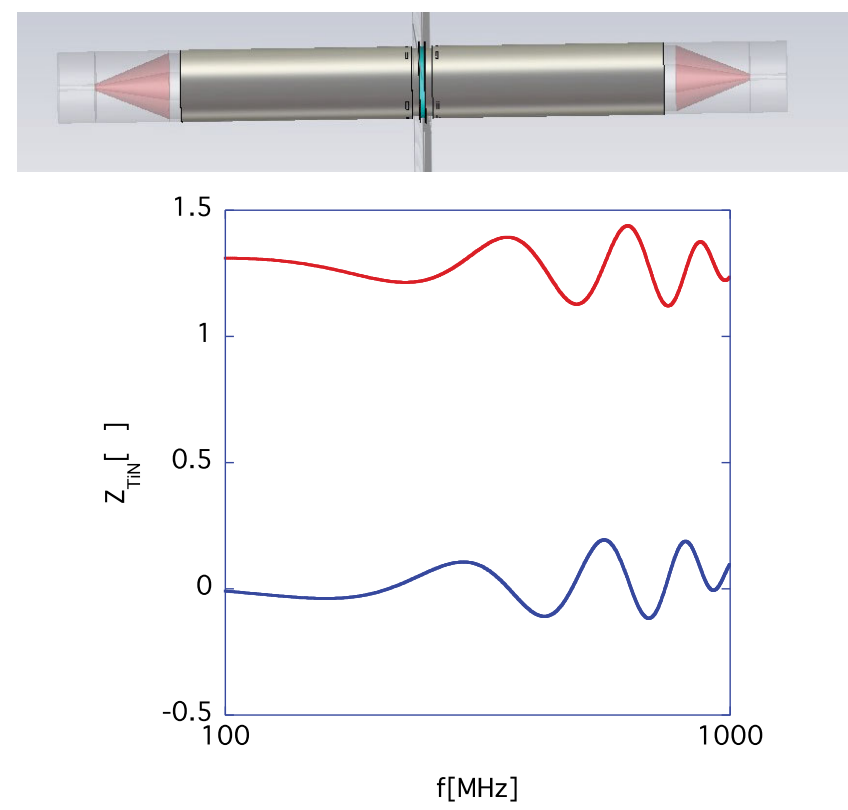

FIG. 9. Simulations (lower) of the longitudinal impedance with both tapers in the setup shown in the upper figure. The horizontal axis is frequency in logarithmic scale.

tapers is below 1.1 up to $1 \mathrm{GHz}$. The simulation result in Fig. 9, where the red and blue lines show the real and imaginary parts of the impedance, illustrates the oscillation behavior with the amplitude $0.2 \Omega$, whose order is the same as that in the measurements in the left figure of Fig. 7. The simulation results indirectly support our expectation that the tapers impose significant constraints on the accuracy of the impedance measurements of the ceramic break with TiN coating.

From the engineering points of view, the TiN coating thickness can vary from $10 \mathrm{~nm}$ to $15 \mathrm{~nm}$ on the ceramic

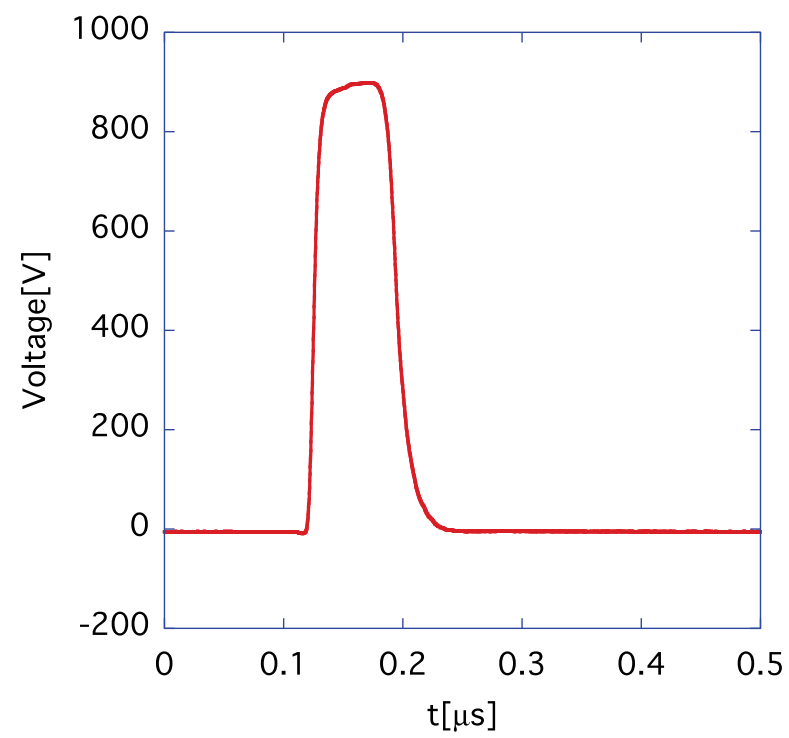

break [25]. Accordingly, the conductivity could be modulated from $\sigma_{\mathrm{TiN}}=2.3 \times 10^{6}[\mathrm{~S} / \mathrm{m}]$ to $\sigma_{\mathrm{TiN}}=3.5 \times 10^{6}[\mathrm{~S} / \mathrm{m}]$ with fixed dc resistance of the TiN coating. However, the main point is that the impedances do not depend on the frequency significantly, as well as it does not follow the resistive wall impedance as $[5,7]$

$Z_{\mathrm{res}}=g Z_{0} \frac{(1+j)}{4 \pi a} \tanh \left[(1+j) \sqrt{\frac{\omega \mu_{0} \sigma_{\mathrm{TiN}}}{2}} t\right] \sqrt{\frac{2 \omega}{c Z_{0} \sigma_{\mathrm{TiN}}}}$,

apparently. After all, we successfully obtained the beam impedance $Z_{\text {cerTiN }, L}$, or the electric impedance $Z_{\mathrm{TiN}}$, which does not significantly depend on the frequency.

Let us now measure the current signal at the downstream port of the chamber, and monitor the signal on the ceramic break. Figure 10 depicts the measurements. The monitored signal on the ceramic break (right) reproduced well the current signal at the downstream port of the chamber (left). The ratio between both maximum values is determined by

$$
\frac{g}{\sigma_{\mathrm{TiN}} 2 \pi a t Z_{50}} \simeq 0.014
$$

which is consistent with Eq. (53).

Here, let us closely investigate whether the measured data satisfies Eq. (51) or not. For this purpose, we calculated the left-hand side of Eq. (51) after the Fourier transformation of the observed results in the time domain. We then compared it with the right-hand side after substituting the observed $S_{12}$ by the network analyzer into the right-hand side of Eq. (51). Figure 11 shows the results. The red and blue lines depict the right-hand side of Eq. (51), while the purple and green lines represent the

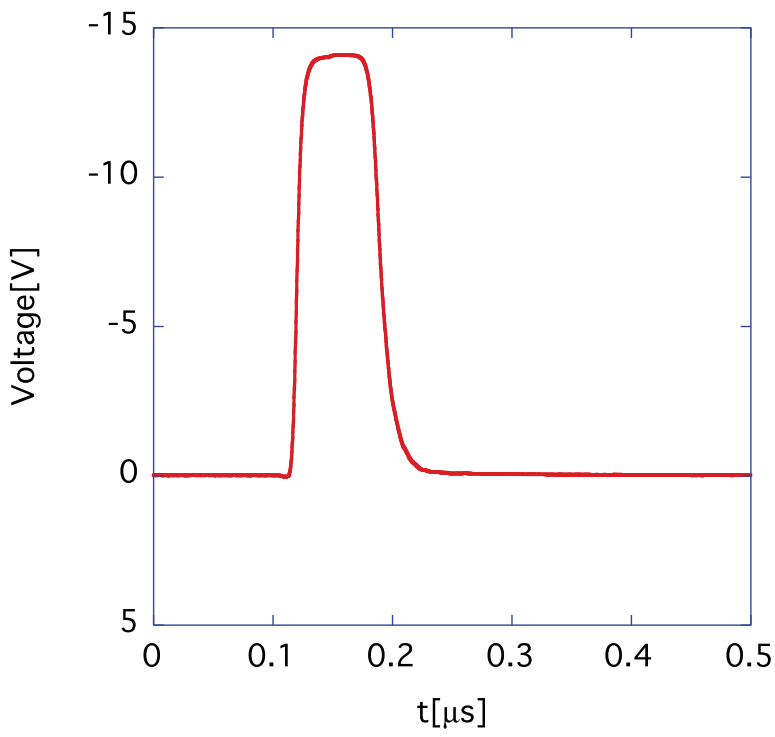

FIG. 10. Current signal at the downstream end of the chamber (left) and the monitored signal on the ceramic break (right). 


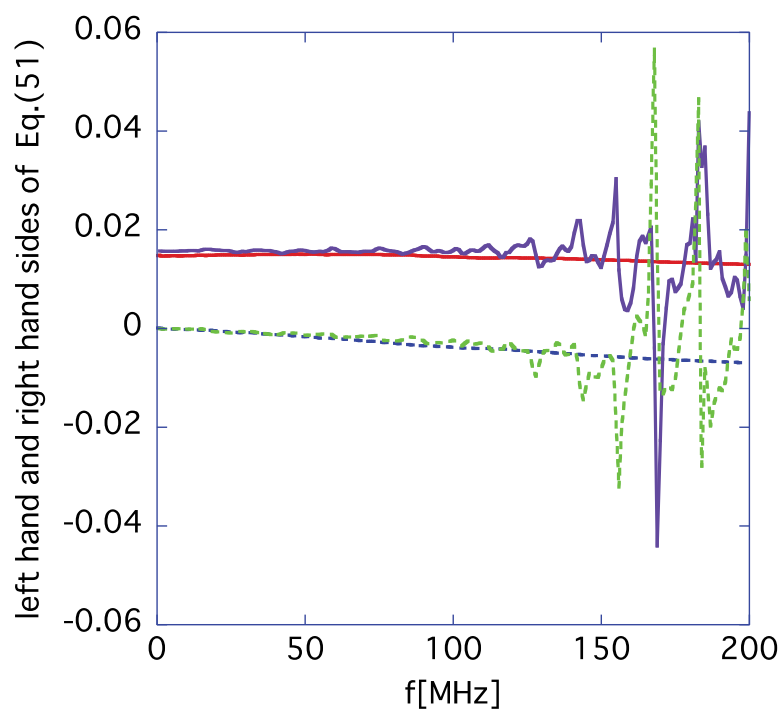

FIG. 11. Comparison between the left- (purple/green) and right-hand (red/blue) sides of Eq. (51) for the ceramic break in the upper figures of Fig. 4.

left-hand side of Eq. (51). The solid and dashed lines represent the real and imaginary parts of Eq. (51). Both results are in good agreement below $100 \mathrm{MHz}$; however, the agreement became worse toward a high frequency because of the lack of accuracy of the measured pulse in the time domain with the oscilloscope.

In this measurement, the bandwidth and the sampling rate were set to be $500 \mathrm{MHz}$ and $5 \mathrm{GS} / \mathrm{s}$, respectively, in the oscilloscope with an 8-bit vertical resolution [17]. The data was averaged 100 times. However, as Fig. 12 shows, the absolute value of the Fourier transformed current pulse $\tilde{V}_{1}$ is drastically reduced beyond around $100 \mathrm{MHz}$, owing to the rising/falling times and the pulse period of the input pulse. That is why the measurement signal to noise ratio limits the significant data below the frequency. In principle, by making the pulse approach the $\delta$-function pulse, we can widen the effective frequency range in the measurement,

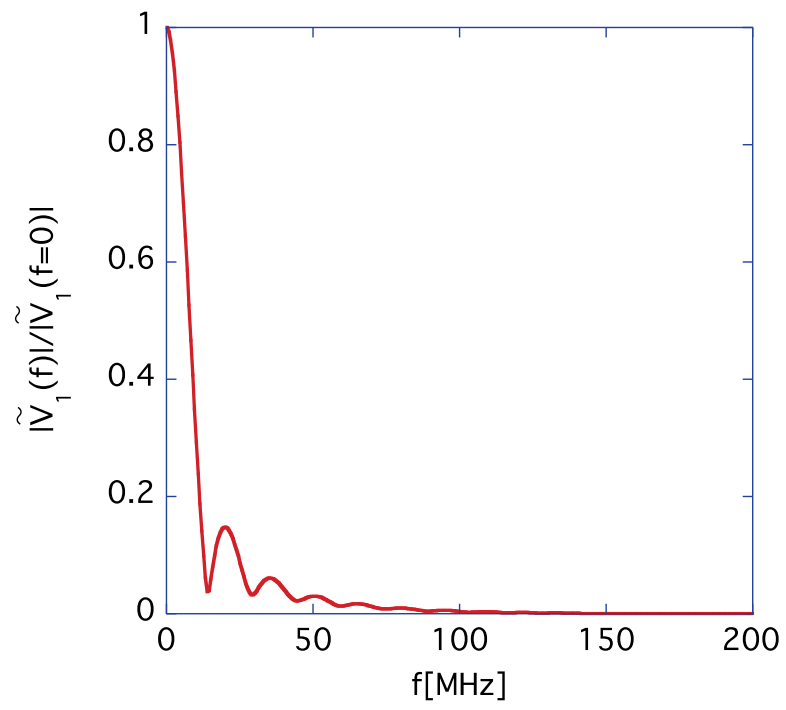

FIG. 12. Normalized Fourier transformed current pulse $\left|\tilde{V}_{1}(f)\right| /\left|\tilde{V}_{1}(f=0)\right|$ (measurement).

though the minimum rising/falling times were restricted to $5 \mathrm{~ns}$ in this pulse generator [14].

Based on the results, we expect that the relation (51) is satisfied at high frequencies; thus, let us directly observe the characteristic of $S_{12}^{(m o n)}$ up to $4.5 \mathrm{GHz}$ using the network analyzer. Figure 13 shows the amplitude (left) and phase (center/right). The right figure is a scaled-up figure of the center figure from 0 to $200 \mathrm{MHz}$. The blue line in the center and right figures shows line: $2 \pi f \sqrt{L C} l / 2$ for reference. The uniqueness of the amplitude and the linearity of the phase were good until around $2.5 \mathrm{GHz}$. The authors previously investigated the characteristic of triangle and concave pentagon electrodes [12] and the exponential electrode until a few $\mathrm{GHz}$ [26]. The characteristic of this monitor utilizing a TiN-coated ceramic is better than that of the cases at a low frequency extreme because the amplitude of $S_{12}^{(\text {mon) }}$ does not rise from zero at $f=0$, which is different
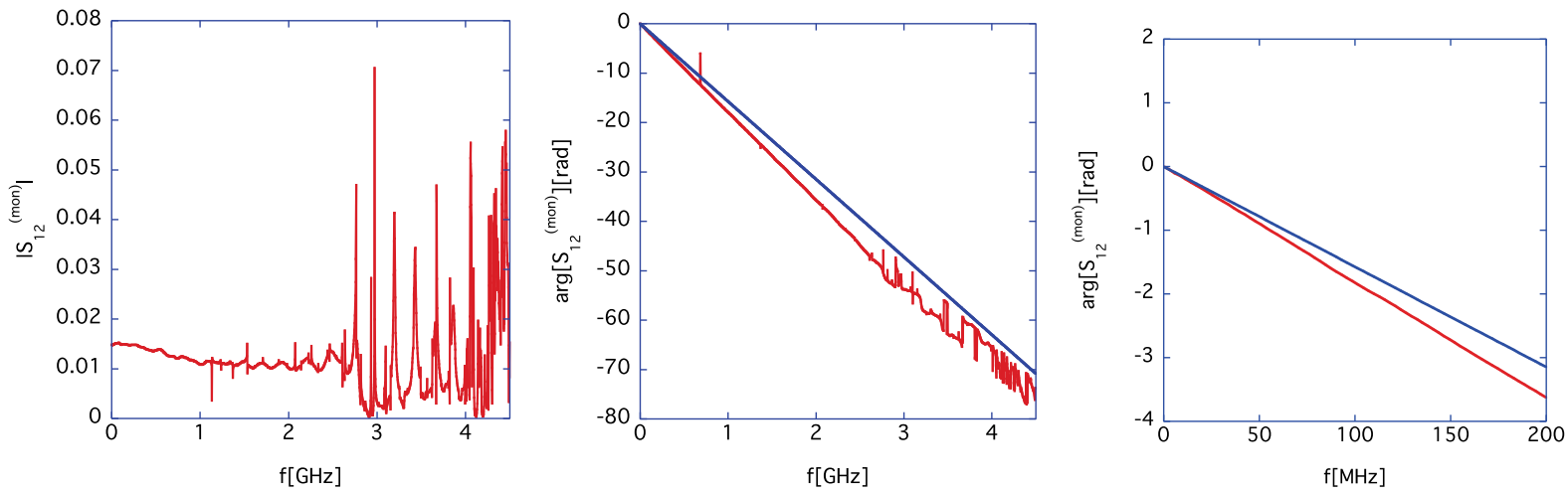

FIG. 13. Amplitude (left) and phase (center/left) of $S_{12}^{(\text {mon) }}$ for the ceramic break in the upper figure of Fig. 4, where the right figure is a scaled-up figure of the center figure from 0 to $200 \mathrm{MHz}$. 

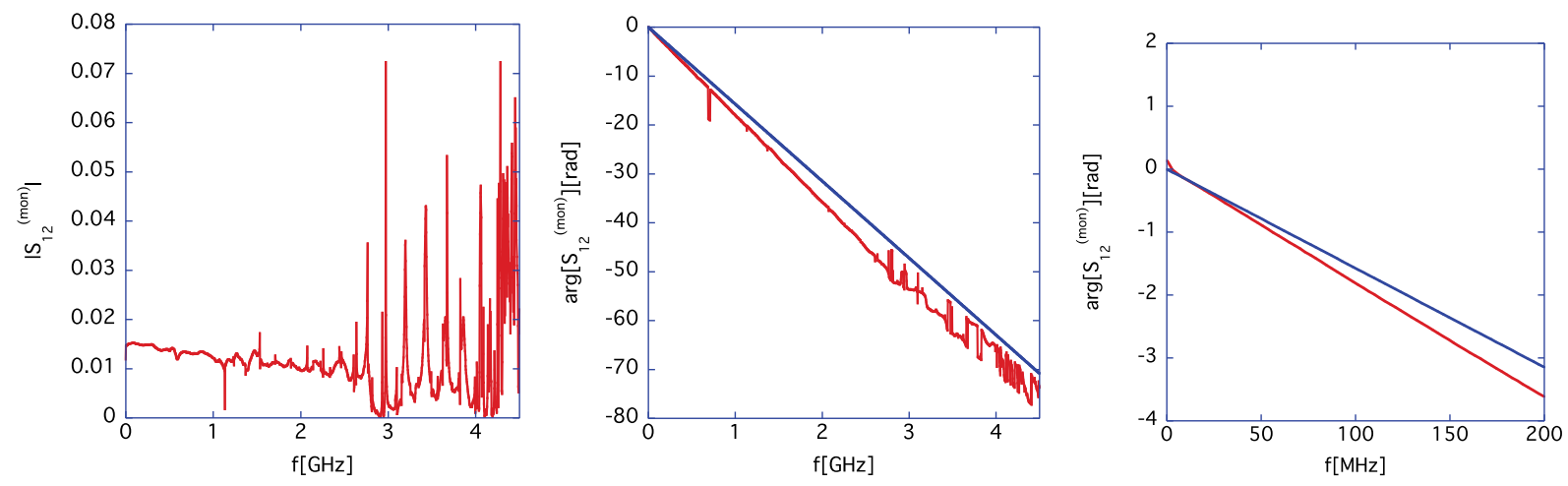

FIG. 14. Amplitude (left) and phase (center/left) of $S_{12}^{(\mathrm{mon})}$ for the ceramic break in the lower figures of Fig. 4, where the right figure is a scaled-up figure of the center figure from 0 to $200 \mathrm{MHz}$.

from that of the triangle and concave pentagon electrodes and the exponential electrode. Consequently, we can directly observe the beam current behavior instead of its derivative using this ceramic break monitor.

Next, let us consider the other ceramic break in the lower figures of Fig. 4 by investigating how the characteristic of the simplest ceramic break discussed earlier is deformed because of the properties of its surroundings.

In the lower figures of Fig. 4, both edges of the ceramic were surrounded (360 degrees) by the metal grounded through the metal stand, which deformed the wall current path. Figure 14 represents the measurements of the amplitude (left) and the phase (center/left) of the $S$-matrix component $S_{12}^{(\mathrm{mon})}$ for the ceramic break, where the right figure is a scaled-up figure of the center figure from 0 to $200 \mathrm{MHz}$. The blue line in the center and right figures depicts line: $2 \pi f \sqrt{L C} l / 2$ for reference. Figure 15 illustrates the combined results of the amplitude (left) and phase (right) of $S_{12}^{(\text {mon })}$ from 0 to $100 \mathrm{MHz}$ for the ceramic breaks in the lower (red) and upper (blue) figures of Fig. 4. Even in the ceramic break in the lower figures of Fig. 4, the uniqueness of the amplitude and the linearity of the phase were relatively good until around $2.5 \mathrm{GHz}$. On the other hand, the red lines in Fig. 15 reveal that the absolute value of $S_{12}^{\text {(mon) }}$ slightly decreased, while its argument slightly enhanced (inductive) at a low frequency, compared to the results for the ceramic breaks in the upper figures of Fig. 4 (blue).

Figure 16 illustrates the effects of the amplitude and the phase of $S_{12}^{(\text {mon })}$ on the monitored pulse in the time domain. Remarkably, the tail of the monitored pulse decreased and gradually recovered to zero after approximately $1 \mu \mathrm{s}$. To clarify that the cause of this phenomenon is the slight modulation of $S_{12}$ around $0 \mathrm{MHz}$ in Fig. 14, let us compare the left- (purple/green) and right-hand sides (red/blue) of Eq. (51), where the left-hand side is obtained by the Fourier

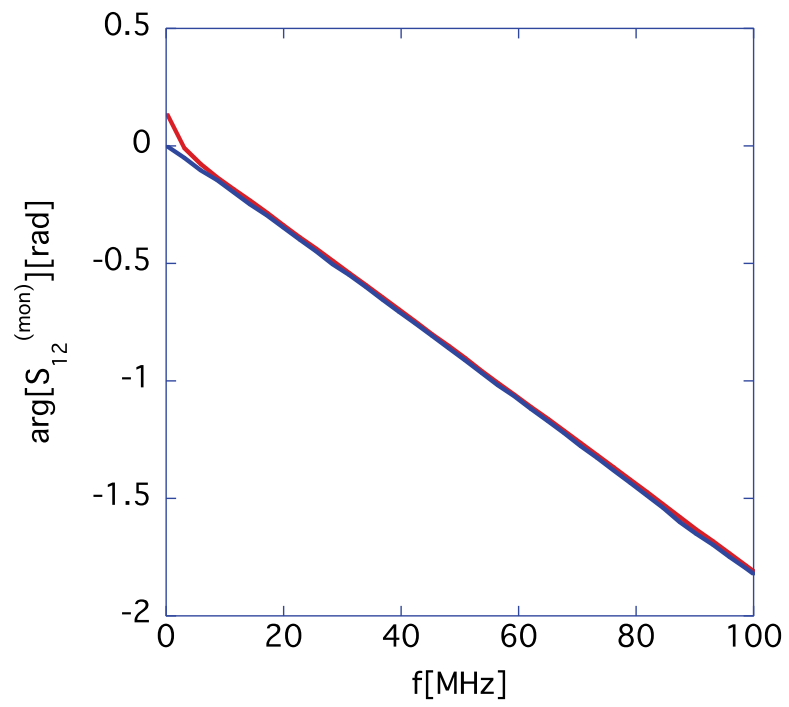

FIG. 15. Amplitude (left) and phase (right) of $S_{12}^{(\mathrm{mon})}$ from 0 to $100 \mathrm{MHz}$ for the ceramic breaks in the lower (red) and upper (blue) figures of Fig. 4. 


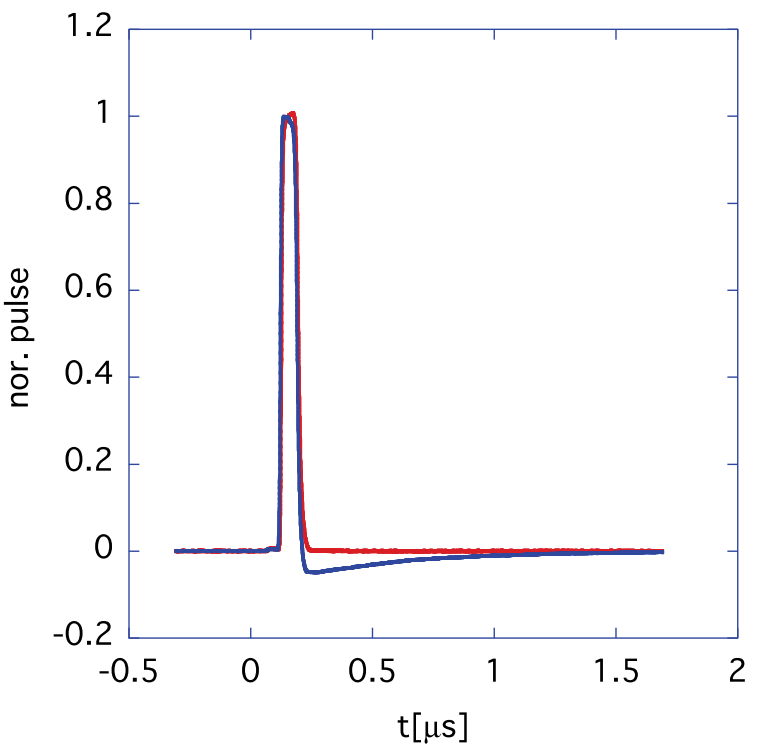

FIG. 16. Current signal at the downstream end (red) of the chamber and the monitored signal (blue) on the ceramic break in the lower figure of Fig. 4. Both maximum amplitudes are normalized to one, for comparison.

transformation of the original pulse and the monitored currents corresponding to the results in Fig. 16, while the right-hand side is obtained using Fig. 14. Figure 17 depicts the results, which demonstrate the validity of Eq. (51) below $100 \mathrm{MHz}$, even for this ceramic break in the lower figures of Fig. 4.

Now, let us parametrize the results $\mathcal{F} / Z_{50}$ by referring to the procedure in Sec. IIIC. The red and blues lines in Fig. 18 represent the real and imaginary parts of the righthand side of Eq. (51), respectively, which correspond to the

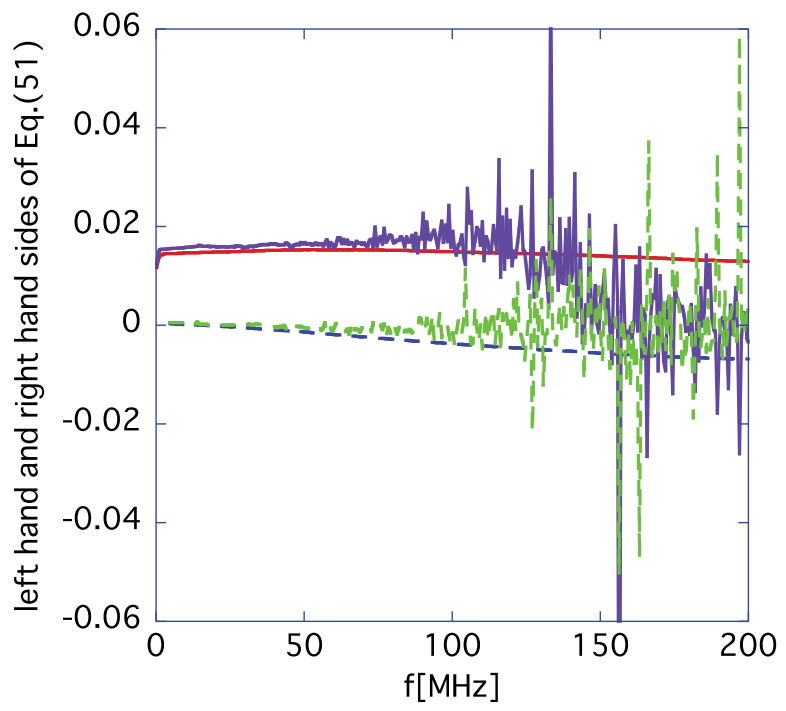

FIG. 17. Comparison between the left- (purple/green) and right-hand (red/blue) sides of Eq. (51) for the ceramic break in the lower figures of Fig. 4.

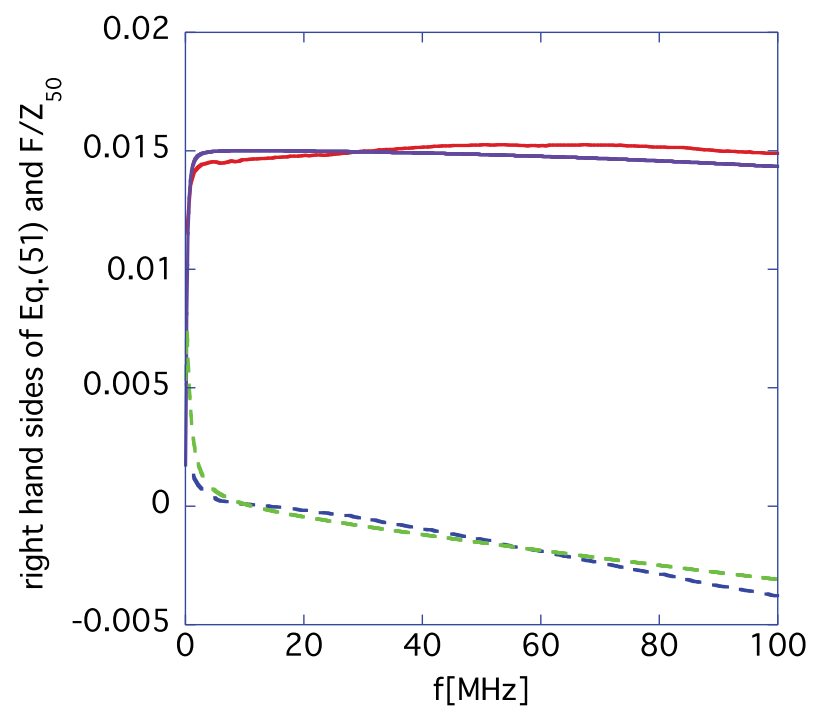

FIG. 18. Comparison between the right-hand side of Eq. (51) (red/blue) and the right-hand side $\mathcal{F} / Z_{50}$ (purple/green) of Eq. (48) for the ceramic break in the lower figures of Fig. 4, where $\mathcal{R}=0.75 \Omega, \mathcal{L}=0.432 \mu \mathrm{H}$, and $\mathcal{C}=0.46 \mathrm{nF}$. The solid and dashed lines show their real and imaginary parts.

scaled-up figure in Fig. 17 at a low frequency. If we choose $\mathcal{R}=0.75, \Omega, \mathcal{L}=0.432 \mu \mathrm{H}$, and $\mathcal{C}=0.46 \mathrm{nF}$ in Eq. (54), the right-hand side of Eq. (48) $\left(\mathcal{F} / Z_{50}\right)$ is parametrized as the purple (real) and green (imaginary) lines in Fig. 18, respectively. Figure 18 illustrates the validity of Eq. (48) at a low frequency through the parametrization of $\mathcal{F}$ in Eq. (48). Figure 19 expresses the analytical results of $\mathcal{V}_{2}(t)$ corresponding to the monitored pulse obtained after $\mathcal{F}$ is substituted into Eq. (57). The red line depicts the

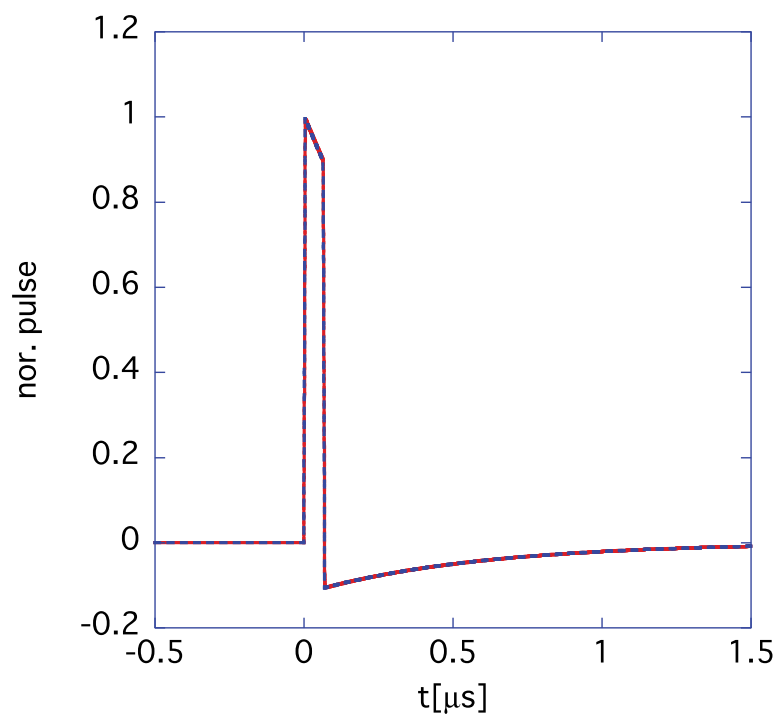

FIG. 19. Normalized results of $\mathcal{V}_{2}(t)$ obtained by analytically inverse Fourier transforming the purple and green lines in Fig. 18. The red line is obtained by Eq. (58), while the blue line is obtained by Eq. (61), neglecting the contribution from $\mathcal{C}$. 


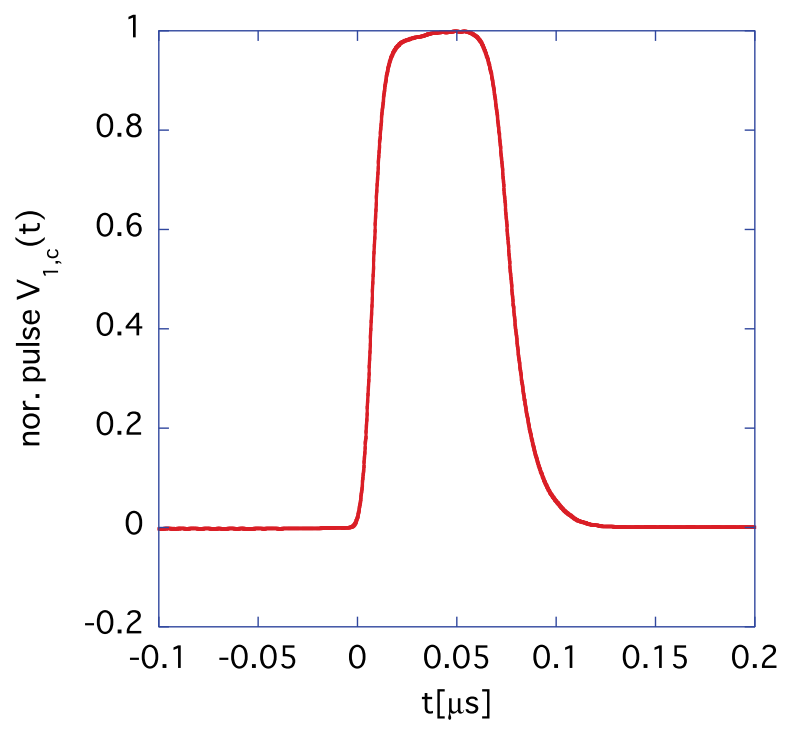

FIG. 20. Reproduced current signal by using the monitored signal.

rigorous result by Eq. (58), while the blue line represents the approximated result by Eq. (61) with the assumption of $\mathcal{C}=0$. The results showed that the difference between both results was negligible, demonstrating that the inductive components stemming from this type of ceramic break deformed the monitored pulse on the blue line from the red one in Fig. 16.

Finally, let us reversely reproduce the original pulse by the monitored pulse by applying Eq. (51). For this purpose, let us calibrate the monitored pulse as

$$
\mathcal{V}_{1, c}(t)=-\int_{-\infty}^{\infty} \frac{d \omega}{2 \pi} \frac{e^{j \omega(t-\sqrt{L C} l)} \tilde{V}_{2}}{S_{12}^{(\mathrm{mon})}} W(\omega)
$$

where $\mathcal{V}_{1, c}(t)$ is the calibrated pulse; $\tilde{V}_{2}$ is the Fouriertransformed monitored voltage originally observed with an oscilloscope; and $W(\omega)$ is the window function. We assumed a Hamming-type window function as follows [27],

$W(\omega)= \begin{cases}0.54-0.46 \cos \left[\pi\left(\frac{\omega}{2 \pi w i n}-1.0\right)\right], & \text { for } 0 \leq \frac{\omega}{2 \pi w i n} \leq 1, \\ 0, & \text { otherwise }\end{cases}$ where, win is the parameter to specify the window chosen as $200 \mathrm{MHz}$ in the trapezoidal beam pulse (with a rising time of $5 \mathrm{~ns}$ ).

Figure 20 shows the calibrated $\mathcal{V}_{1, c}(t)$ for the ceramic break in the lower figure of Fig. 4, reproducing the red line in Fig. 16. The drop of the signal at the tail of the pulse and its recovery of it on the blue line in Fig. 16 were successfully eliminated from the result.

\section{SUMMARY}

Nature tries to minimize the energy loss of the beam; hence, the entire wall current continues to run in the thin TiN even when the skin depth is much larger than the chamber thickness, except the ceramic break with extremely thin TiN coating (typically smaller than a few $\mathrm{nm})$. In this study, the impedance of the ceramic break with TiN coating consisted of three components in parallel: resistive wall term caused by TiN coating, radiation term, and capacitive term made by the ceramic itself. This characteristic is useful in developing a wall current monitor with an improved frequency response.

Considering some drawbacks of the numerical simulation approach, we mainly investigated herein the characteristic from the theoretical and measurement points of view. As a result, this study demonstrated this feature of the monitor up to a few $\mathrm{GHz}$ after clarifying the relation (51) between the current (beam) pulse and the monitored signal in the frequency domain.

The uniqueness of the amplitude and the linearity of the phase of $S_{12}^{(\text {mon) }}$ were good until around $2.5 \mathrm{GHz}$. Reversely, the application of relation (51) was useful in calibrating the monitored signal to reproduce the original current signal. Moreover, the characteristic of this monitor was better than those of the triangle and concave pentagon electrodes and the exponential electrode at the low frequency extreme because the $S_{12}^{\text {(mon) }}$ amplitude did not rise from zero at the low frequency in this ceramic break monitor.

\section{ACKNOWLEDGMENTS}

This work was supported by JSPS KAKENHI Grant No. JP17K05124. The authors also would like to thank all members of J-PARC project at JAEA/KEK.

$$
\begin{aligned}
& \text { APPENDIX: CONCRETE EXPRESSIONS OF } \boldsymbol{A}_{\mathbf{0}}^{(\boldsymbol{m})}, \boldsymbol{C}_{\mathbf{0}}^{(\boldsymbol{m})}, \boldsymbol{I}_{\mathbf{0}}^{(\boldsymbol{m})}, \boldsymbol{K}_{\mathbf{0}}^{(\boldsymbol{m})},\langle\boldsymbol{\alpha}\rangle, \\
& \langle\langle\boldsymbol{\alpha}\rangle\rangle_{\boldsymbol{m}},\langle\boldsymbol{J}\rangle,\langle\langle\boldsymbol{J}\rangle\rangle_{\boldsymbol{m}},\left\langle\left\langle\boldsymbol{J}^{(\boldsymbol{n})}\right\rangle\right\rangle_{\boldsymbol{m}},\langle\boldsymbol{Y}\rangle,\langle\langle\boldsymbol{Y}\rangle\rangle_{\boldsymbol{m}}, \text { AND }\left\langle\boldsymbol{Y}^{(\boldsymbol{n})}\right\rangle_{\boldsymbol{m}} \\
& A_{0}^{(m)} \simeq \mu_{m}^{\epsilon} a\left[I_{0}^{\prime}\left(\mu_{m}^{\epsilon} a\right) K_{0}\left(\mu_{m}^{\epsilon} a_{2}\right)-I_{0}\left(\mu_{m}^{\epsilon} a_{2}\right) K_{0}^{\prime}\left(\mu_{m}^{\epsilon} a\right)\right] \cosh \kappa_{\mathrm{TiN}} t \\
& -\frac{j Z_{0}\left(\mu_{m}^{\epsilon}\right)^{2} a \sigma_{\mathrm{TiN}}\left[I_{0}\left(\mu_{m}^{\epsilon} a_{2}\right) K_{0}\left(\mu_{m}^{\epsilon} a\right)-I_{0}\left(\mu_{m}^{\epsilon} a\right) K_{0}\left(\mu_{m}^{\epsilon} a_{2}\right)\right] \sinh \kappa_{\mathrm{TiN}} t}{k \beta \epsilon^{\prime} \kappa_{\mathrm{TiN}}}
\end{aligned}
$$




$$
\begin{aligned}
& C_{0}^{(m)} \simeq \frac{\mu_{m}^{\epsilon} a\left[I_{0}^{\prime}\left(\mu_{m}^{\epsilon} a\right) K_{0}\left(\mu_{m}^{\epsilon} a_{2}\right)-I_{0}\left(\mu_{m}^{\epsilon} a_{2}\right) K_{0}^{\prime}\left(\mu_{m}^{\epsilon} a\right)\right] \kappa_{\mathrm{TiN}} \sinh \kappa_{\mathrm{TiN}} t}{\sigma_{\mathrm{TiN}}} \\
&-\frac{j Z_{0}\left(\mu_{m}^{\epsilon}\right)^{2} a\left[I_{0}\left(\mu_{m}^{\epsilon} a_{2}\right) K_{0}\left(\mu_{m}^{\epsilon} a\right)-I_{0}\left(\mu_{m}^{\epsilon} a\right) K_{0}\left(\mu_{m}^{\epsilon} a_{2}\right)\right] \cosh \kappa_{\mathrm{TiN}} t}{k \beta \epsilon^{\prime}}, \\
& I_{0}^{(m)} \simeq \frac{j k \beta a \epsilon^{\prime}\left[I_{0}^{\prime}\left(\mu_{m}^{\epsilon} a\right) K_{0}^{\prime}\left(\mu_{m}^{\epsilon} a_{2}\right)-I_{0}^{\prime}\left(\mu_{m}^{\epsilon} a_{2}\right) K_{0}^{\prime}\left(\mu_{m}^{\epsilon} a\right)\right] \cosh \kappa_{\mathrm{TiN}} t}{Z_{0}} \\
&+\frac{\mu_{m}^{\epsilon} a \sigma_{\mathrm{TiN}}\left[I_{0}^{\prime}\left(\mu_{m}^{\epsilon} a_{2}\right) K_{0}\left(\mu_{m}^{\epsilon} a\right)-I_{0}\left(\mu_{m}^{\epsilon} a\right) K_{0}^{\prime}\left(\mu_{m}^{\epsilon} a_{2}\right)\right] \sinh \kappa_{\mathrm{TiN}} t}{\kappa_{\mathrm{TiN}}}, \\
& K_{0}^{(m)} \simeq \frac{j k \beta a \epsilon^{\prime}\left[I_{0}^{\prime}\left(\mu_{m}^{\epsilon} a\right) K_{0}^{\prime}\left(\mu_{m}^{\epsilon} a_{2}\right)-I_{0}^{\prime}\left(\mu_{m}^{\epsilon} a_{2}\right) K_{0}^{\prime}\left(\mu_{m}^{\epsilon} a\right)\right] \kappa_{\mathrm{TiN}} \sinh \kappa_{\mathrm{TiN}} t}{\sigma_{\mathrm{TiN}} Z_{0}} \\
&+\mu_{m}^{\epsilon} a\left[I_{0}^{\prime}\left(\mu_{m}^{\epsilon} a_{2}\right) K_{0}\left(\mu_{m}^{\epsilon} a\right)-I_{0}\left(\mu_{m}^{\epsilon} a\right) K_{0}^{\prime}\left(\mu_{m}^{\epsilon} a_{2}\right)\right] \cosh \kappa_{\mathrm{TiN}} t \\
& \mu_{m}^{\epsilon}=\sqrt{\frac{m^{2} \pi^{2}}{4 w^{2}}-k^{2} \beta^{2} \epsilon^{\prime}}
\end{aligned}
$$

for $t \ll\left(a_{2}-a\right)$, the prime on the modified Bessel functions denotes the derivative with their arguments, and

$$
\begin{aligned}
& \langle\alpha\rangle=\frac{\beta c I_{1}(\bar{k} \sigma)}{\pi \sigma \bar{k} a I_{0}(\bar{k} a)} \frac{\sin k w}{k w}, \\
& \langle\langle\alpha\rangle\rangle_{m}=-\frac{\beta c I_{1}(\bar{k} \sigma)}{\pi \sigma \bar{k} a I_{0}(\bar{k} a)} \frac{k\left[(-1)^{m} e^{-j k w}-e^{j k w}\right]}{j\left(k^{2}-\frac{m^{2} \pi^{2}}{4 w^{2}}\right)}, \\
& \langle J(z)\rangle=-\sum_{s=1}^{\infty} \frac{2 \pi a}{w b_{s}^{2}}-\sum_{s=1}^{\infty} \frac{\pi a^{2}\left(e^{-j 2 \frac{b_{s}}{a} w}-1\right)}{j w^{2} b_{s}^{3}}, \\
& \langle\langle J(z)\rangle\rangle_{m}=\sum_{s=1}^{\infty} \frac{\pi j\left(1+(-1)^{m}\right)\left(e^{-j 2 \sqrt{k^{2} \beta^{2}-\frac{j_{0 . s}^{2}}{a^{2}} w}}-1\right)}{w a\left(k^{2} \beta^{2}-\frac{j_{0, s}^{2}}{a^{2}}-\frac{m^{2} \pi^{2}}{4 w^{2}}\right) \sqrt{k^{2} \beta^{2}-\frac{j_{0, s}^{2}}{a^{2}}}},
\end{aligned}
$$

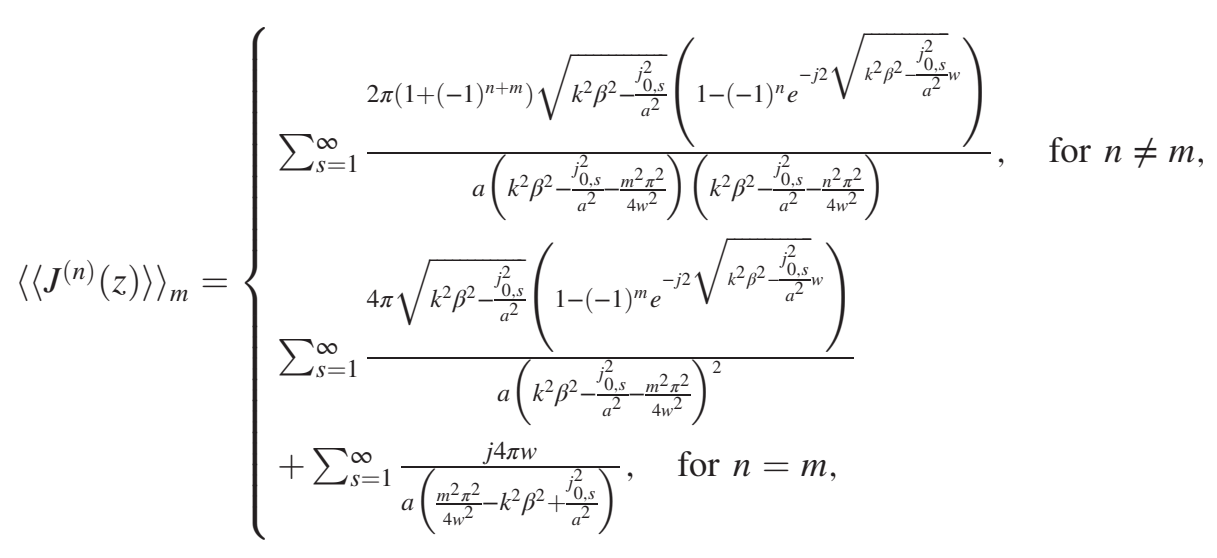




$$
\begin{aligned}
& \langle Y(z)\rangle=-\int_{0}^{\infty} d \zeta \frac{2}{w \pi a_{2} \zeta\left(k^{2} \beta^{2}+\frac{\zeta}{a_{2}^{2}}\right) H_{0}^{(1)}\left(e^{j \frac{\pi}{2}} \sqrt{\zeta}\right) H_{0}^{(2)}\left(e^{j \frac{\pi}{2}} \sqrt{\zeta}\right)} \\
& -\int_{0}^{\infty} d \zeta \frac{\left(e^{-j 2 w} \sqrt{k^{2} \beta^{2}+\frac{\zeta}{a_{2}^{2}}}-1\right)}{j w^{2} \pi a_{2} \zeta\left(k^{2} \beta^{2}+\frac{\zeta}{a_{2}^{2}}\right)^{\frac{3}{2}} H_{0}^{(1)}\left(e^{j \frac{\pi}{2}} \sqrt{\zeta}\right) H_{0}^{(2)}\left(e^{j \frac{\pi}{2}} \sqrt{\zeta}\right)} \\
& \langle\langle Y(z)\rangle\rangle_{m}=-\int_{0}^{\infty} d \zeta \frac{j\left(1+(-1)^{m}\right)\left(1-e^{-j 2 \sqrt{\frac{\zeta}{a_{2}^{2}}+k^{2} \beta^{2}} w}\right)}{w \pi a_{2} \zeta \sqrt{\frac{\zeta}{a_{2}^{2}}+k^{2} \beta^{2}}\left(\frac{\zeta}{a_{2}^{2}}+k^{2} \beta^{2}-\frac{m^{2} \pi^{2}}{4 w^{2}}\right) H_{0}^{(1)}\left(e^{\frac{\pi}{2} j} \sqrt{\zeta}\right) H_{0}^{(2)}\left(e^{\frac{\pi}{2} j} \sqrt{\zeta}\right)},
\end{aligned}
$$

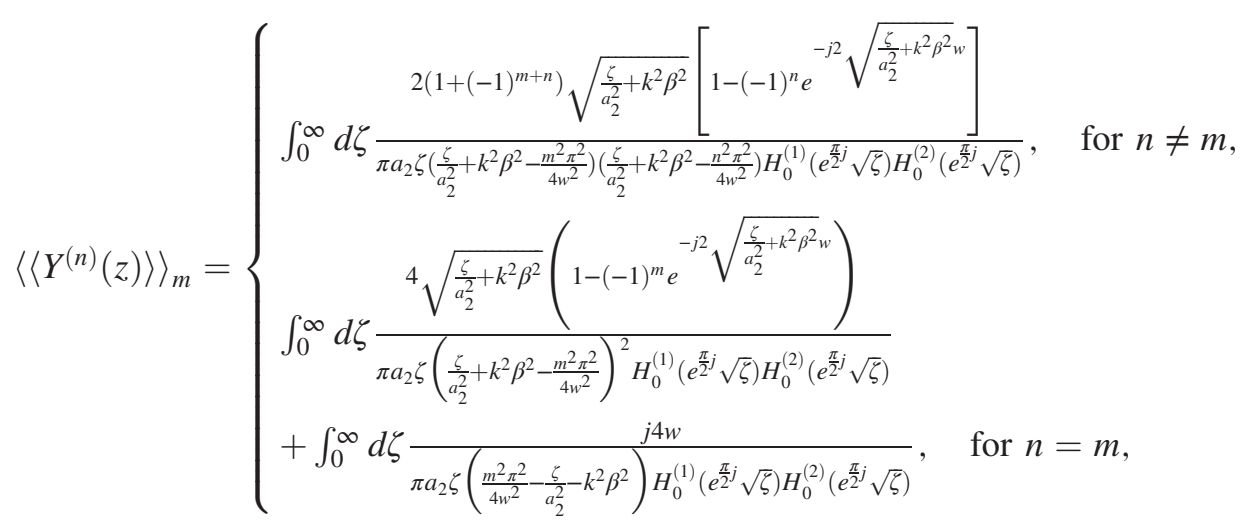

$H_{m}^{(1)}(z)$ and $H_{m}^{(2)}(z)$ are the Hankel function of the first and the second kinds, respectively, $m$ and $n$ are positive integers, $b_{s}^{2}=k^{2} \beta^{2} a^{2}-j_{0, s}^{2}=-\beta_{s}^{2}$, and $b_{s}$ approaches $-j \beta_{s}$ for $j_{0, s}>k \beta a$. Although it looks like $\zeta=$ $m^{2} \pi^{2} a_{2}^{2} / 4 w^{2}-k^{2} \beta^{2} a_{2}^{2}$ is a singular point in some integrands, it disappears after summarizing the relevant terms.

[1] Y. Shobuda, Y. Irie, and S. Igarashi, Analytical method for the evaluation of field modulation inside the rf-shielded chamber with a time-dependent dipole magnetic field, Phys. Rev. Accel. Beams 12, 032401 (2009).

[2] K. Ohmi, T. Toyama, and C. Ohmori, Electron cloud instability in high intensity proton rings, Phys. Rev. Accel. Beams 5, 114402 (2002).

[3] K. Ohmi, T. Toyama, and C. Ohmori, Erratum: Electron cloud instability in high intensity proton rings, Phys. Rev. Accel. Beams 6, 029901(E) (2003).

[4] Y. H. Chin, S. Lee, K. Takata, T. Toyama, Y. Shobuda, and H. Tsutsui, Impedance generated by a ceramic chamber with rf shields and tin coating, in Proceedings of HB2006, 125, Tsukuba, Japan (2006), https://inspirehep .net/literature/739642.

[5] A. W. Chao, Physics of Collective Beam Instabilities in High Energy Accelerators, (Wiley, New York, 1993).
[6] B. W. Zotter and S. A. Kheifets, Impedances and Wakes in High-Energy Particle Accelerators, (World Scientific, Singapore, 1998).

[7] Handbook of Accelerator Physics and Engineering, edited by A. W. Chao and M. Tigner (World Scientific, Singapore, 1999).

[8] http://j-parc.jp/index-e.html.

[9] Y. Shobuda, Y. H. Chin, and K. Takata, Coupling impedances of a gap in vacuum chamber, Phys. Rev. Accel. Beams, 10, 044403 (2007).

[10] Y. Shobuda, Y. H. Chin, and K. Takata, Coupling impedances of a resistive insert in a vacuum chamber, Phys. Rev. Accel. Beams, 12, 094401 (2009).

[11] Y. Shobuda, Y. H. Chin, and K. Takata, Impedance of a ceramic break and its resonance structures, Phys. Rev. Accel. Beams, 17, 091001 (2014).

[12] Y. Shobuda, Y. H. Chin, K. Takata, T. Toyama, and K. Nakamura, Triangle and concave pentagon electrodes for an improved broadband frequency response of stripline beam position monitors, Phys. Rev. Accel. Beams Beams, 19, 021003 (2016).

[13] M. Abramowitz and I. Stegun, Handbook of Mathematical Functions-with Formulas, Graphs, and Mathematical Tables (Dover Pub., New York, 1974), http://people .math.sfu.ca/ cbm/aands/intro.htm.

[14] https://www.tek.com/Tektronix, KEITHLEY 3390 $50 \mathrm{MHz}$ Arbitrary Waveform Generator. 
[15] https://www.digikey.com/product-detail/en/directed-energyinc/PVM-1001-P/2145-PVM-1001-P-ND/10268386.

[16] www.matsusada.co.jp, Matsusada Precision Inc., HJPM1R15-SP

[17] https://www.tek.com/ Tektronix, DPO 5054 Digital Photosphor Oscilloscope

[18] http://www.tmele.jp/english/, Tamagawa Electronics Co. Ltd, UFA-100NPJ-20

[19] Agilent Technologies, Now, Keysight Technologies, https://www.keysight.com/jp/ja/home.html.

[20] H. Hahn and F. Pedersen, On coaxial wire measurements of the longitudinal coupling impedance, Brookhaven National Lab., Upton, NY (USA), Report No. BNL50870, 1978, https://www.osti.gov/servlets/purl/.

[21] H. Hahn, Validity of coupling impedance bench measurements, Phys. Rev. Accel. Beams, 3, 122001 (2000).

[22] R. L. Gluckstern and R. Li, Analysis of coaxial wire measurement of longitudinal coupling impedance, Part. Accel. 29, 159 (1990), https://inspirehep.net/literature/286587.
[23] T. Kroyer, F. Caspers, and E. Gaxiola, Longitudinal and transverse wire measurements for the evaluation of impedance reduction measures on the MKE extraction kickers, CERN, Geneva, Report No. AB-Note-2007-028, http:// cdsweb.cern.ch/record/1035461/files/ab-note-2007-028 .pdf.

[24] CST STUDIO SUITE, http://www.cst.com.

[25] J. Kamiya (private communication).

[26] T.P. R. Linnecar, The high frequency longitudinal and transverse pick-Ups in the CERN SPS accelerator, CERN Report No. CERN-SPS-ARF-SPS/78/17, 1979, https://cds .cern.ch/record/119558? ln=ja; T. P. R. Linnecar, The high frequency longitudinal and transverse pick-Ups in the CERN SPS accelerator, IEEE Trans. Nucl. Sci. 26, 3409 (1979).

[27] G. Harris, On the use of windows for harmonic analysis with the discrete Fourier transform, , Proc. IEEE 66, 51 (1978). 